\title{
Type 2 tasks in the theory of signal detectability: Discrimination between correct and incorrect decisions
}

\author{
SUSAN J. GALVIN \\ University of Otago, Dunedin, New Zealand \\ JOHN V. PODD \\ Massey University, Palmerston North, New Zealand \\ and \\ VIT DRGA and JOHN WHITMORE \\ Victoria University, Wellington, New Zealand
}

\begin{abstract}
It has been known for over 40 years that there are two fundamentally different kinds of detection tasks in the theory of signal detectability. The Type 1 task is to distinguish between events defined independently of the observer; the Type 2 task is to distinguish between one's own correct and incorrect decisions about those Type 1 events. For the Type 1 task, the behavior of the detector can be summarized by the traditional receiver operating characteristic (ROC) curve. This curve can be compared with a theoretical ROC curve, which can be generated from overlapping probability functions conditional on the Type 1 events on an appropriate decision axis. We show how to derive the probability functions underlying Type 2 decisions from those for the Type 1 task. ROC curves and the usual measures of performance are readily obtained from those Type 2 functions, and some relationships among various Type 1 and Type 2 performance measures are presented. We discuss the relationship between Type 1 and Type 2 confidence ratings and caution against the practice of presenting transformed Type 2 ratings as empirical Type 1 ratings.
\end{abstract}

It was a dark and stormy night. A lone psychophysicist hurried up the slope of the beach to the shelter of the bush, the right angle of her tracks in the sand recording her abrupt change in direction. She pulled her cell phone from her map pocket, dialed quickly, and held the phone under the hood of her parka.

"Dunedin Police."

"Hi, I'm on Tautuku Beach in the Catlins, and I think I just saw an emergency flare over the head at the south end."

"Are you sure you're right about that?"

"Not really. The weather is a mess here. I'm pretty sure I saw a red light arc through the sky, but there are houses on the peninsula, and I suppose it could have come from there. But it seemed too far away for that. Anyway, I didn't want

This article is based in part on the masters dissertations of J.V.P. (1975) and S.J.G. (1988). The derivation of the general equations for Type 2 probability functions was presented at the 1987 meeting of the New Zealand Psychological Society, Dunedin, New Zealand. We thank Carl Beuke, Karen Dobkins, Stephen Link, Kenneth Malmberg, Jeff Miller, Robert O'Shea, Amy Rezec, Tom Robinson, Maria Schuchinsky, Larry Thibos, James Townsend, Jonathan Victor, and an anonymous reviewer for comments on earlier manuscripts and discussions about Type 2. Correspondence concerning this article should be addressed to S. J. Galvin, Department of Psychology, University of Otago, P. O. Box 56, Dunedin, New Zealand (e-mail: galvin@ @sy.otago.ac.nz). John T. Wixted was editor. to read about a wreck with all hands lost in the Times tomorrow and then wish I'd called."

"Righto. I'll tell Balclutha to get their woollies on. Stay on the line, please."

How good are people at telling when their decisions are right or wrong? This skill is an important factor in learning from experience and predicting the consequences of our choices. The ability to assess one's decisions is the target of research in specialist fields, such as transport safety, medical diagnosis, witness reliability, and interpersonal relationships. It is surprising, then, that such an important metacognitive faculty has rarely been analyzed using the criterion-free measures developed within the theory of signal detectability (TSD).

The task of discriminating between one's own correct and incorrect decisions was first described and named the Type 2 task in TSD by Clarke, Birdsall, and Tanner (1959) and Pollack (1959). In a Type 1 task, an observer decides which of two or more events defined independently of the observer has occurred (e.g., deciding that there was a flare or that there was not a flare, in our introductory example). In this article, we consider only Type 1 choices between two events, and we refer to these generically as signal $(S)$ and noise $(N)$ events. At the time a Type 1 decision is made, another event occurs: The observer is either correct $(C)$ or incorrect $(I)$. The task of discriminating between one's own correct and incorrect Type 1 decisions is called a Type 2 
task (Clarke et al., 1959; Pollack, 1959). The only fundamental difference between the two tasks lies in the kind of events to be discriminated.

For either the Type 1 or the Type 2 task, the basis for discriminating between the two events is the decision axis. The decision axis is the evidence associated with the events, or some transformation of the evidence, such as the likelihood ratio, used to make the decisions. For each task, two probability functions ${ }^{1}$ show how values of the decision axis are distributed conditional on the occurrence of each of two events ( $S$ and $N$ for Type 1 , and $C$ and $I$ for Type 2 ).

The appropriate pair of conditional probability functions can be used to generate theoretical receiver operating characteristic (ROC) curves for each task. The conventional Type 1 ROC curve is a plot of the probability that an observer will report that a signal has occurred when it has occurred against the probability that the observer will report a signal event has occurred when it has not occurred, for each possible criterion applied to the decision axis. The Type 1 ROC curve illustrates an observer's ability to discriminate one Type 1 event from another. The Type 2 ROC curve is a plot of the probability that an observer will report a Type 1 decision as correct when it was correct against the probability that the observer will report a decision as correct when it was not correct for each possible criterion applied to the Type 2 decision axis. The Type 2 ROC curve illustrates an observer's ability to discriminate one Type 2 event from another.

Although Clarke et al. (1959) described and named the Type 2 task over 40 years ago, their classification was not accompanied by the development of a general mathematical theory enabling a comparison of empirical results with theoretical predictions. In this article, we show how the ability to discriminate correct from incorrect is related to the ability to discriminate signal from noise and discuss the implications of this relationship for psychological research.

Although there have been no theoretical curves with which to compare them, empirical Type 2 ROC curves do exist in the literature. We begin by describing some of this research. We then formally define Type 1 and Type 2 tasks and show how to compute discrete theoretical Type 2 probability functions from theoretical Type 1 functions for the dice game of Swets, Tanner, and Birdsall (1961). We generalize this procedure by deriving a set of formulae that can be applied to any pair of Type 1 probability functions (i.e., those conditional on events $S$ and $N$ ) of some decision variable to produce Type 2 probability functions (conditional on the events $C$ and $I$ ) of that variable. We show how to use transformations of these Type 2 functions to produce Type 2 probability functions for other decision axes (such as likelihood ratio). We also present six corollaries to the Type 2 theory that highlight some interesting properties of Type 2 ROC curves and measures of Type 2 performance. We conclude by considering how Type 1 and Type 2 confidence ratings are related.

\section{A Brief History of Type 2 Decisions}

The problem of Type 2 decisions grew out of an attempt to apply TSD to research on communication between a source and a receiver (Egan \& Clarke, 1956; Egan, Clarke, \& Carterette, 1956; Pollack \& Decker, 1958). In these sourcereceiver experiments, one person, the source, would read a word out loud over a noisy channel. The receiver would select, from a prelearned list of words, the word thought to have been read out and then would give a rating of confidence that the identification had been correct. Pollack (1959) and Clarke et al. (1959) realized that two fundamentally different kinds of tasks were being required of the observer: One was to discriminate between (or among) Type 1 events, and one was to assess, trial by trial, one's own correctness. Pollack's main concern was to distinguish between measures of sensitivity for the two tasks, whereas Clarke et al. attempted to formulate a mathematical theory that would incorporate the differences between the tasks, as well as make clear the appropriate usage of measures of sensitivity. Clarke et al. posed the problem of producing a pair of Type 2 probability functions for a likelihood ratio ideal observer whose Type 1 task was based on a pair of Gaussian distributions with equal variance. They derived a pair of Type 2 probability functions that were not the solution for the likelihood ratio observer but were the probability functions for another Type 2 decision axis. Their derivation did, however, make it clear that Type 1 probability functions can and should be used to generate Type 2 functions. We will consider their theory in Appendix A.

Since 1959, the Type 2 classification has appeared in some studies of memory but has been largely unused for four decades, with Clarke et al. (1959) attracting a brief mention in textbooks as a sort of a curiosity (e.g., Green \& Swets, 1966; Macmillan \& Creelman, 1991; Murdock, 1974; Vickers, 1979). Macmillan and Creelman did note that "although the analysis of Clarke et al. is almost as old as the standard Type-1 method, it is little used. This is unfortunate, for many experiments that are unsuitable for Type 1 are susceptible in principle to Type-2 description"(p. 82). Type 2 decisions have been used, however, since the early days of experimental psychology in the context of such resurgent topics as calibration theory (Keren, 1988, 1991; Peirce \& Jastrow, 1884) and perception without awareness (Galvin, Beuke, Keogh, \& Robinson, 2000; Kolb \& Braun, 1995; Kunimoto, Miller, \& Pashler, 2001; Weiskrantz, 1986, 1998).

Type 2 tasks have also had a significant presence in the literature because of the popular technique of taking Type 2 ratings and converting these into what we will call here pseudo-Type 1 ratings. If, for example, a choice between events $S$ and $N$ is followed by a rating on a 4-point scale, an 8-point pseudo-Type 1 scale can be created by assigning ratings of 1 to 4 following an $N$ decision to the ratings -4 to -1 and assigning ratings of 1 to 4 following an $S$ decision to ratings 1 to 4 (e.g., Norman \& Wickelgren, 1969). The rationale for this seems to be that both forms of questioning tap the same representation of confidence. We will discuss the wisdom of this once the theory has been elaborated.

\section{Type 2 ROC Analysis in the Study of Memory}

In the 1960s and 1970s, ROC analysis featured in the debate between supporters of incremental learning and 
those of all-or-none learning. McFadden and Greeno (1966) had observers perform recall and recognition tests for the third letter in a three-letter stimulus. They compared observers' performance in two tests using the same items, to see whether items remembered incorrectly on the first test could be remembered on the second test; if they were, it would indicate partial learning for those items. Each individual did two recall tests, two recognition tests, or a recall test followed by a recognition test. The observers followed each test response with a rating of their confidence that their decision had been right. McFadden and Greeno gave a very clear explanation of the difference between Type 1 and Type 2 tasks and used the confidence ratings about the correctness of the Type 1 decisions to construct Type 2 ROC curves. They found that although recognition performance was generally better than recall, Type 2 analysis showed that the observers were better able to discriminate their correct and incorrect recall decisions than their correct and incorrect recognition decisions. These results provided an early demonstration that good Type 1 performance does not necessarily imply good Type 2 performance.

Unfortunately, the early attempts to clarify the problem of Type 2 decisions did not prevent some confusion about the nature of Type 2 ROC analysis. Healy and Jones (1973) noted that, in particular, there arose a tendency to consider any confidence rating to be a Type 2 task. In fact, either a Type 1 task or a Type 2 task can be done using either a confidence rating scale or a binary choice. The difference between Type 1 and Type 2 tasks lies in which events are being evaluated, not in whether the evaluation is binary or a rating. An example of this confusion can be found in a paper by Banks (1970). He asked observers to learn a list of words and then presented them with a series of test words, some from the list and some new. Observers responded to each test word with a rating on a five-category scale ranging from certain it was on the list to certain it was not on the list. This is a Type 1 task, not a Type 2 task, because the observers were rating their certainty that the word was on the study list and were choosing between the events old and new. ${ }^{2}$ However, Banks wrote that "The ROC constructed from confidence ratings is termed the Type II ROC" ( p. 84), but he indicated no source for this definition. It is clear he was not claiming to coin the term Type II, since he mentioned the paper by Clarke et al. (1959) as pertinent to the question of how to treat Type II ROC curves. It is possible that Banks associated the term Type II with rating experiments because some of the earliest papers in which ROC curves were derived from ratings were based on Type 2 data. Banks referred to one such paper, Pollack and Decker (1958), as his model for this analysis. Pollack and Decker's ROC curves are Type 2 because the observers are asked to give ratings that increase with increasing confidence in the correctness of their word identifications and the ratings are tallied separately for correct and incorrect word identifications in order to generate Type 2 hit rates and false alarm rates. It is not the fact that these ROC curves are based on ratings that makes them Type 2.
Another possible confusion could arise if an observer were asked to give a binary choice between signal and noise and to follow this with a rating of confidence that the trial was a signal trial. The fact that the second decision is a rating and follows a binary Type 1 decision does not make it a Type 2 decision. If the second decision is a rating of confidence in the signal event, rather than in the correctness of the first decision, then it is a Type 1 rating, no matter when it occurs.

Even when observers have performed what is clearly a Type 2 task and the data have been correctly analyzed to give measures of Type 2 performance, authors have found it difficult to interpret these measures. Murdock (1966) attempted to use Type 2 ratings of correctness to evaluate criterion shifts in a Type 1 task in which subjects were required to recall a word from a list of previously learned word pairs that would match a word read out by an experimenter. Although he noted that the measures of discriminability and criterion were derived from a Type 2 analysis, he, nonetheless, claimed that his results, "show rather clearly that there are criterion changes in STM" (p. 323). He claimed this without acknowledging that they were not the Type 1 criterion changes he had intended to examine. Healy and Jones (1973), in responding to Murdock, correctly pointed out that "values of $d^{\prime}$ and shifts in criteria revealed by Type 2 analysis reflect only [the] evaluation process, not the subject's memory state" (p. 336).

More recently, Critchfield (1993) has used a close analogy of the Type 2 task to assess the self-reports made by students about a delayed-match-to-sample task. The students had to identify which stimulus from a set of test stimuli belonged to a previously presented set of to-beremembered stimuli and to report their choices within $800 \mathrm{msec}$. They then judged whether they had made the correct choice and reported it in time. Critchfield was interested in students' bias toward thinking themselves correct, and correctly interpreted his bias measure in terms of Type 2, not Type 1, discrimination. Higham (2002) also used a Type 2 analysis to study bias, using an observer's choice not to respond at all to a test item as an indication of low confidence in the response they had generated (but not reported). In neither study did the nature of the Type 1 task lend itself to the analysis we present here, but both authors recognized the ability of TSD measures to yield separate information about discriminability and bias in a Type 2 task.

\section{Type 2 Decisions and Calibration in Decision Theory}

Our general theory of Type 2 decisions is related to studies of calibration, which assess whether observers' confidence in the occurrence of an event, reported as a probability that the event occurred, matches the proportion of times the event actually occurred in the set of trials ascribed each probability. A graph of the observers' confidence plotted against the actual proportions is called a calibration curve and can reveal interesting discrepancies between observers' confidence and their actual performance (Keren 1988, 1991; Lichtenstein, Fischhoff, \& 
Phillips, 1982). Studies of calibration require observers to either (1) state their confidence that a designated event occurred, or will occur, or that a statement is true, in a series of trials, or (2) rate their confidence that they were correct after making a binary choice. These are called the full-range task and the half-range task, respectively (Ferrell \& McGoey, 1980; Lichtenstein et al., 1982). The purpose of the full-range task is to see whether observers can achieve correspondence between the gauged occurrence of an event and its actual occurrence. The purpose of the half-range task is to see how well observers believed they performed in a binary choice task. In our TSD context, the full-range task is similar to a Type 1 rating task, and the half-range task is similar to a Type 1 binary choice followed by a Type 2 rating task. In neither case do calibration curves provide measures of an observer's discrimination, but the ratings could be used to generate ROC curves in cases in which the task is worded appropriately. Hosseini and Ferrell (1982) contributed a useful discussion of the relation between calibration and the objective measures of Type 2 ROC analysis in their paper recommending Type 2 (without calling it that) performance as a measure of the insight of students into their own knowledge development.

\section{Type 2 Decisions and the Measurement of Perception Without Awareness}

The Type 2 task has recently taken its place in the psychometric armory of those interested in perception without awareness. Agreeing on a measure of awareness has proved to be a significant challenge to cognitive scientists (for an interesting review, see Merikle, Smilek, \& Eastwood, 2001). Kunimoto et al. (2001) argued that a subjective report of awareness is inadequate and that an objective measure is required. They proposed that observers who can discriminate between signal and noise but cannot discriminate between their own incorrect and correct decisions exhibit perception without awareness. Kunimoto et al. had observers follow a Type 1 decision with a binary confidence report, which they used to calculate $d^{\prime}$ by defining high confidence on correct trials as hits and high confidence on incorrect trials as misses. They were able to demonstrate an ability to identify a signal event (better-thanchance Type 1 performance) at a signal-to-noise ratio that yielded only chance discrimination between correct and incorrect trials (chance Type 2 performance) and classed this as subliminal perception. The rationale for this is that if the observers can do the Type 1 task, but not the Type 2 task, then they do not have conscious access to the evidence used to do the Type 1 task when they give their Type 2 responses.

The criterion for perception without awareness proposed by Kunimoto et al. (2001) has the virtues of reflecting a subjective quality (confidence) and the rigor of objective psychophysics. However, various models of Type 2 performance generated using our theory show that even when Type 1 and Type 2 decisions are based on the same evidence, performance in the two tasks is typically not the same; the area under the Type 1 ROC curve often exceeds the area under the Type 2 ROC curve. In general, therefore, it is unwise to interpret a difference between Type 1 and Type 2 performance as showing that the part of the brain supporting the discrimination has access to one piece of evidence but that the part of the brain supporting visual awareness does not. Galvin et al. (2000) suggested that, in general, to produce evidence of perception without awareness, performance in the Type 2 task should be compared with a prediction of Type 2 performance based on Type 1 performance, rather than with Type 1 performance itself. Corollary 6 of our theory shows that such a prediction can be made regardless of the form of the Type 1 probability functions or of the Type 1 criterion used. We will revisit Kunimoto et al.'s study in the Discussion section, because it provides an interesting case in which the comparison between Type 1 and Type 2 performances is warranted when Type 2 performances is at chance, given a set of limiting assumptions.

Kolb and Braun (1995) used Type 2 tasks to demonstrate perception without awareness of regions of the visual field with different visual texture from that of their surrounds. In the key condition of their experiment, these regions were masked by the presence of opposite motion (in the case of texture defined by motion direction) or by binocular rivalry (in the case of texture defined by line orientation). They obtained Type 2 ROC curves by requiring their observers to indicate which quadrant carried the anomalous texture and then to rate their confidence that they were correct on a 10-point scale. Although the Type 1 performance was very good and similar for the two textures, they found very good Type 2 performance for unmasked stimuli but near-chance Type 2 performance for the masked stimuli. They interpreted this as reflecting "defective" conscious experience (p. 336). Like Kunimoto et al. (2001), Kolb and Braun recognized the value of the Type 2 task of TSD as a way to produce an objective measure of awareness. Without a formal theory of Type 2 decisions, however, they did not realize that a direct comparison of Type 1 and Type 2 ROC curves could be inappropriate. We will turn to such a formal theory now.

\section{The Type 2 Task as an Extension of the Fundamental Detection Problem}

The essence of a Type 2 task is to assess the correctness of a Type 1 decision that has just been made. A Type 2 task may follow any of several kinds of Type 1 task (e.g., single interval or multipleinterval, two event or multiple event). In this article, we present an analysis based on a singleinterval or yes-no choice between two Type 1 events, which will eventually provide the basis for analyses of other forms of Type 2 tasks.

Figure 1 illustrates the Type 2 task as an extension of the fundamental detection problem (FDP), which is the cornerstone of the theory of signal detectability (Birdsall, 1955; Egan, 1975; Swets et al., 1961). The Type 1 task is to indicate whether a signal event, $S$, or a noise event, $N$, has occurred during the clearly marked observation interval. The events $S$ and $N$ occur with prior probabilities $P(S)$ and $P(N)$, respectively. Some value, $x$, of a random vari- 


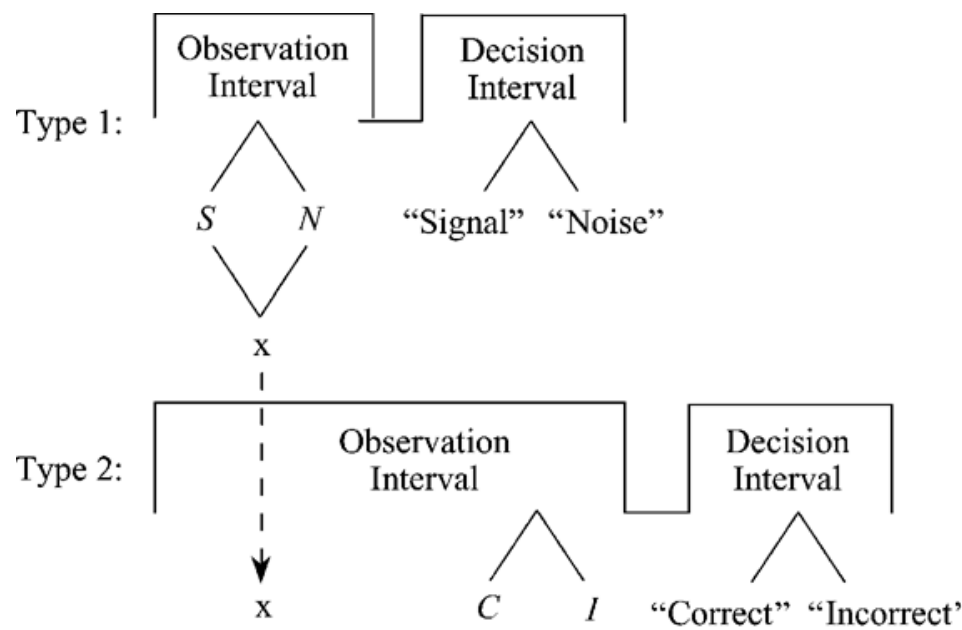

Time $\longrightarrow$

Figure 1. The binary Type 2 task as an extension of the fundamental detection problem. During the Type 1 observation interval, there occurs either the signal event $(S)$ or the noise event $(N)$, along with a value, $x$, of the evidence variable, $X$. In the Type 1 decision interval, the observer must respond either "Yes, a signal occurred" or "No, a signal did not occur." The observation and decision intervals for the Type 1 task together become the observation interval for the Type 2 task, because one of the events correct $(C)$ or incorrect $(I)$ occurs when the observer makes the Type 1 decision and the same value of the evidence, $x$, is used to evaluate whether the Type 1 decision was correct. The observer states either "Yes, it was correct" or "No, it was not correct" during the Type 2 decision interval. Ratings can be used, rather than binary choices, in either task, but the Type 2 task makes sense only if it follows a binary Type 1 task.

able, $X$, is associated with each observation interval, and uncertainty arises about which event has occurred because at least some of the values of evidence can occur as a result of either event. The values of $X$ make up an ordered decision axis and are referred to as values of the stimulus, or evidence. Note that some authors use the term stimulus to refer to whatever was presented to the observer by the experimenter and the term evidence to refer to the variable the observers actually use to make decisions after the stimulus has undergone transformations in their sensory systems. We use the terms stimulus and evidence interchangeably, in the tradition of Egan, because we are not offering a theory of signal transduction and sensory processing but, rather, are analyzing the degree to which pairs of events are discriminable. ${ }^{3}$ Note also that the Type 1 task in the FDP is not to identify the stimulus, as it was in the old source-receiver experiments, but to identify the event that gave rise to the stimulus.

We assume for now that to make a Type 1 decision, an observer adopts some criterion, $k$, on the decision axis $X$; we will address the use of other Type 1 decision axes later. On any trial, the observer applies the following decision rule: Say "Yes, the event $S$ occurred" if $x$ exceeds $k$; otherwise, say "No, it was the $N$ event."

The observation interval and the decision interval for the Type 1 task together may be seen as the observation in- terval for the Type 2 task, because one of the events $C$ or I occurs when the observer makes the Type 1 decision. Although, in general, the Type 1 decision in the FDP can be either binary or a multipoint rating task, a meaningful Type 2 decision can be made only if the Type 1 task that precedes it is binary, because the observer must be either right or wrong for the event $C$ or the event $I$ to have occurred. During the decision interval for the Type 2 task, the observer decides whether $C$ or $I$ has occurred, using the same evidence, $x$, as that used to make the Type 1 decision. The random variable, $X$, provides sufficient evidence for the Type 2 decision because $x$, along with the criterion, $k$, determines the decision made in the Type 1 task. ${ }^{4}$ If $X$ is used as the decision axis for the Type 2 decision, another criterion on $X, m$, is established for that decision. This criterion may or may not be the same as $k$. For the Type 2 task, the observer will say "Yes, my decision was correct" if $x$ exceeds $m$ but will say "No, my decision was incorrect" if $x$ is less than or equal to $m . .^{5}$ Observers may use a Type 2 decision axis other than $X$. If so, all the values of $X$ must map onto the decision axis (call it $W$ ). The Type 2 decision is then made by setting a single criterion on $W$.

It might appear that the Type 2 task is strange indeed, for two reasons. First, it may seem odd to decide on any occasion that a Type 1 decision is incorrect; this seems to amount to changing one's mind in the brief period be- 
tween making the two decisions. However, the case of the drenched psychophysicist provides an example in which that choice would be rational. She noted that her Type 1 criterion was low because of the high cost (loss of life) of not calling the police if the evidence was produced by a flare. She knew that she might well be wrong about the flare and might have chosen "Incorrect" if asked to make a binary Type 2 decision, using a criterion not affected by the outcomes of deciding whether to make the call. The criteria for the Type 1 and the Type 2 decisions may be placed according to very different sets of contingencies. Even without extreme payoffs, observers will soon realize, with explanation and feedback, that answering "I was correct" on every trial will result in an inability to discriminate correct from incorrect.

It is also the case that an experimenter can avoid the apparent paradox of reporting a decision just made as incorrect by using some other labels for the possible responses in a Type 2 task. Unless one is interested in observers' calibration, the actual values of confidence assigned to binary choices or to ratings are irrelevant to the job of measuring how well observers can discriminate correct from incorrect decisions. All that matters is that the labels be ordinal and assigned in the correct order and that the observers understand that correctness is being assessed. Type 2 binary choices between "low confidence that I was correct" and "high confidence that I was correct" are quite legitimate. In a Type 2 rating task a set of criteria, $m_{1}, m_{2}$, $m_{3}, \ldots$, divides the decision axis into regions that will produce ratings of confidence that the Type 1 decision was correct. This set could have "I was unsure I was right" at one end of the scale and "I was sure I was right" at the other.

The second feature of the description of the Type 2 task we have given so far that may be disconcerting to some is the use of $X$ as the Type 2 decision axis. Consider the following example, in which the observer would be unlikely to use $X$ as the Type 2 decision axis. The observer's Type 1 task is to say whether an adult picked at random from the population of New Zealand is male (event $M$ ) or female (event $F$ ), on the basis of that adult's height. Imagine that the probability distributions of the evidence (height) conditional on the events $M$ and $F$ can be approximated by normal distributions with equal variance, with the mean of the distribution conditional on $M$ being somewhat higher than the mean of the distribution conditional on $F$. Assume, also, that there are equal numbers of men and women in New Zealand. A rational Type 1 decision rule might be to respond "Male" if the person is $170 \mathrm{~cm}(5 \mathrm{ft} 7$ in) or taller and "Female" otherwise. Given that Type 1 criterion, it is difficult to come up with a sensible Type 2 decision rule with a single criterion applied to $X$ as the decision axis. The Type 2 probability functions in this case will not be normal distributions, as we will show in an example later. The probability function of $X$ conditional on the event $C$ will be bimodal, and an observer will be most confident of decisions based on very large or very small heights. Using a single Type 2 criterion on the $X$-axis re- quires the response "incorrect" when the heights are very small, because they will fall below all but an extremely low Type 2 criterion. ${ }^{6}$ A transformation of $X$ will be necessary to allow the observer to respond "highly confident" about decisions based on both very large and very small heights. Using the likelihood ratio of the Type 2 distributions would allow this and would provide a model of optimal performance. Another possible Type 2 decision axis is $W=|X-k|$, the difference between the Type 1 criterion and the evidence. The reason we concentrate on $X$ as the Type 2 decision axis is that to derive models for decision axes that are transformations of $X$, we first derive the Type 2 probability functions of $X$ and then produce the Type 2 distributions for other decision axes by using the standard method of deriving distributions of transformations of random variables. We give a worked example of this for a pair of discrete Type 1 probability functions below and an example for a pair of continuous probability functions in Appendix A. Egan (1975) gave several useful examples of transformations of decision variable in his Appendix B.

Note also that we are not assuming that observers use $X$ as their decision axis in the Type 1 tasks. Researchers who have developed a theory of the transformation of $X$ that observers may use to make Type 1 decisions in their experimental context can substitute the transformed variable, $V=$ $t_{1}(X)$, for $X$ in the derivation that follows.

We reiterate this important point to emphasize that the formulae we derive can be used in any context for which an experimenter already has a model for Type 1 decision making: By deriving the probability functions for the case in which $X$ has been used as decision axis for both Type 1 and Type 2 tasks, we do not mean to suggest that observers will typically use $X$ for either task. Our aim is to provide a way to derive probability functions of some decision variable conditional on $C$ and $I$ when the probability functions of that decision variable conditional on $S$ and $N$ are known. Transformations of variables before and after this step are the responsibility of the user! Although we present examples of Type 2 models to illustrate our derivations, none of these should be interpreted as our theory of how observers approach specific tasks. Our goal is to enable readers to generate their own models for Type 2 tasks and to understand how to use them.

We proceed now by introducing notation for the probability functions and ROC curves for Type 1 and Type 2 tasks. For the Type 1 task, the random variable $X$ has probability functions $f(x \mid S)$ and $f(x \mid N)$ conditional on the events $S$ and $N$, respectively; ${ }^{7}$ the corresponding cumulative distribution functions of $X$ are $F(x \mid S)$ and $F(x \mid N) .{ }^{8}$ The hit rate $\left(H R_{1}\right)$ and false alarm rate $\left(F A R_{1}\right)$ for the Type 1 task, given that the criterion used is $k$, are given by

$$
H R_{1}=1-F(x \mid S)
$$

and

$$
F A R_{1}=1-F(x \mid N) .
$$

The plot of $H R_{1}$ against $F A R_{1}$ for all criteria, $k$, on the $X$ axis is the Type 1 ROC curve. ${ }^{9}$ 
From $f(x \mid S)$ and $f(x \mid N)$, another pair of probability functions of $x, f_{k}(x \mid C)$, and $f_{k}(x \mid I)$, conditionalon $C$ and $I$, can be derived for each value of $k$. We refer to these as Type 2 probability functions of $X$. Each pair of Type 2 functions has corresponding cumulative distribution functions $F_{k}(x \mid C)$ and $F_{k}(x \mid I)$. The hit rate $\left(H R_{2}\right)$ and false alarm rate $\left(F A R_{2}\right)$ for the Type 2 task, given that the Type 1 criterion was $k$ and the Type 2 criterion was $m$, are given by

$$
H R_{2}=1-F_{k}(m \mid C)
$$

and

$$
F A R_{2}=1-F_{k}(m \mid I) .
$$

The Type 2 ROC curve for a particular pair of probability functions $f_{k}(x \mid C)$ and $f_{k}(x \mid I)$ is the plot of $H R_{2}$ against $F A R_{2}$ for each possible criterion, $m$, on the $X$ axis. The number of Type 2 ROC curves that can be derived from a pair of Type 1 probability functions is equal to the number of distinct criteria that may exist for the Type 1 task. (This will be an infinite number for continuous Type 1 probability functions.) The reason for this will become clear in the computational example below.

Type 2 ROC curves can also be derived for the case in which the likelihood ratio of the probability functions $f_{k}(x \mid C)$ and $f_{k}(x \mid I), l_{k}(X)=f_{k}(x \mid C) / f_{k}(x \mid I)$, or any other transformation of $X$, is used as the Type 2 decision axis. The Type 2 probability functions of $l_{k}(X), g_{k}\left[l_{k}(x) \mid C\right]$ and $g_{k}\left[l_{k}(x) \mid I\right]$, are obtained by applying the transformation $l_{k}(X)=f_{k}(x \mid C) / f_{k}(x \mid I)$ to the probability functions $f_{k}(x \mid C)$ and $f_{k}(x \mid I)$. (We give an example of this in Ap- pendix A, discussing the theory of Clarke et al., 1959.) The Type 2 ROC curve for this task is then defined by

$$
H R_{2}=1-G_{k}(\alpha \mid C)
$$

and

$$
F A R_{2}=1-G_{k}(\alpha \mid I),
$$

where $G_{k}\left[l_{k}(x) \mid C\right]$, and $G_{k}\left[l_{k}(x) \mid I\right]$, are the cumulative distribution functions corresponding to $g_{k}\left[l_{k}(x) \mid C\right]$ and $g_{k}\left[l_{k}(x) \mid I\right]$ and $\alpha$ is the value of $l_{k}(X)$ used as the criterion in the Type 2 decision.

\section{Type 2 ROC Curves: A Computational Example}

Swets et al. (1961) and Green and Swets (1966) used the example of the dice game to illustrate how to produce an ROC curve. We use the same device to show how to compute a pair of Type 2 probability functions. In the dice game, the experimenter throws three dice (hidden from the observer's gaze) and reports only the sum of the digits on the three upturned faces. Two of the dice are ordinary, but the third has a 0 printed on three sides and a 3 printed on the other three sides. The observer's task on each trial is to use the sum to say whether the strange die has landed with a three facing up (the $S$ event has occurred) or a zero facing up (the $N$ event has occurred).

Figure 2 shows how the possible values of the evidence (the sums) are distributed conditional on the $S$ and $N$ events. The light gray bars show that for the $N$ event (the odd die shows zero), the most common sum is 7 and that the sums can range from 2 to 12 . The probability function

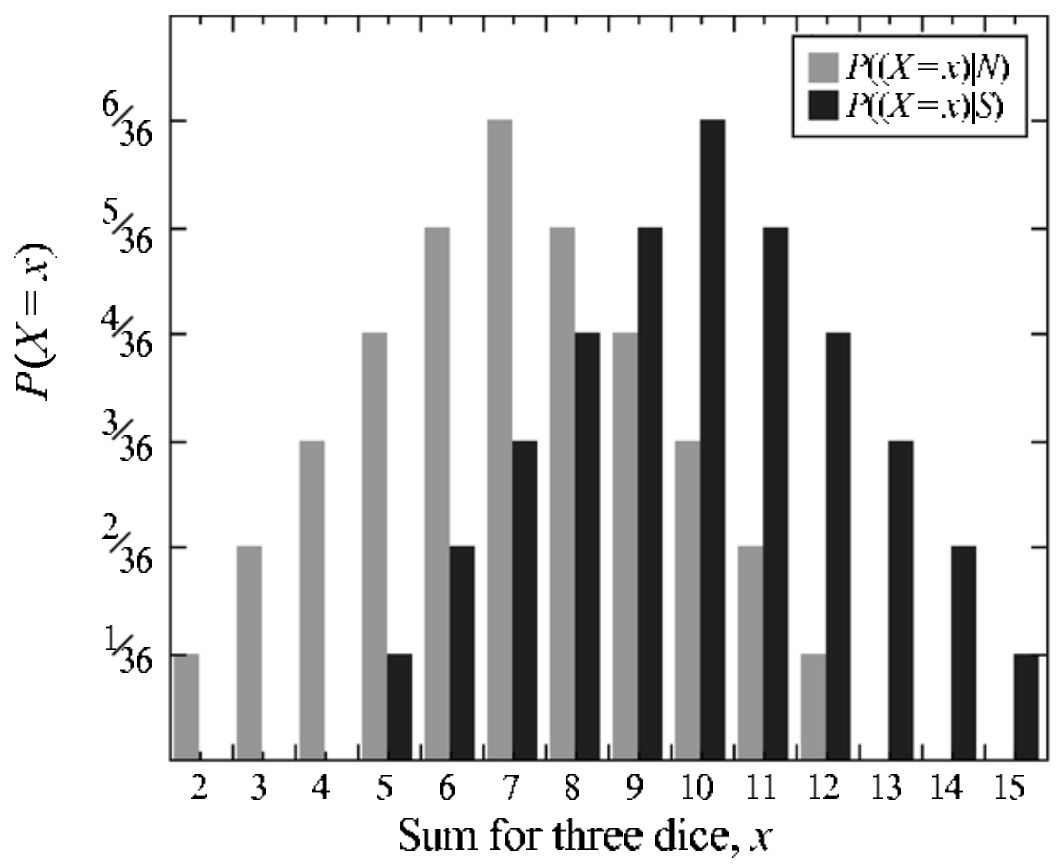

Figure 2. Distributions of the sum of three dice conditional on the $N$ event (third die shows 0 ) and the $S$ event (third die shows 3 ) in the dice game. 
for the $S$ event has the same shape as that for the $N$ event but is shifted three to the right. One can obtain an ROC curve by plotting the observer's hit rate and false alarm rate for every possible criterion value, where a hit is saying that the odd die showed a 3 when it did show a 3 and a false alarm is saying that it showed a 3 when it actually showed a 0 . That ROC curve is shown as the solid line in Figure 3.

Columns 2 and 3 in Table 1 show the Type 1 probability functions plotted in Figure 2. The first step in deriving the Type 2 distributions is to calculate the joint probabilities for the evidence and each event. Because the selection of the event for any trial is independent of the selection of the evidence value from the appropriate distribution, the joint probabilities are just the product of the two probabilities, and these are shown in the last two columns of Table 1.

At this point, we must assume a Type 1 criterion in order to proceed. Any one will do, but readers of Green and Swets (1966) and other keen persons will have realized that the proportion of trials correct will be maximized by choosing a criterion of 8 , saying "signal" if the experimenter reports a sum of 9 or greater and "noise" if the sum is 8 or less, so we will start with that. The dotted horizontal line in Table 1 marks this Type 1 criterion. The shaded probabilities are for event-evidence combinations that yield correct decisions; the rest produce incorrect decisions. For example, if the $N$ event occurs but the evidence value 11 turns up, the observer must say "signal," because 11 is greater than 8 .

In Table 2 (columns 2 and 3), the joint probabilities have been sorted according to whether each particular combination of event and evidence will produce a correct decision or an incorrect one. The shaded probabilities from Table 1 are listed together under the heading "probability that $X$ takes the value $x$ and the correct response is made, given $k=8$," or $P_{8}[(X=x) \cap C]$. The other probabilities make up $P_{8}[(X=x) \cap I]$, giving the joint probabilities in column 2 . The sum of the probabilities in the $P_{8}[(X=x)$

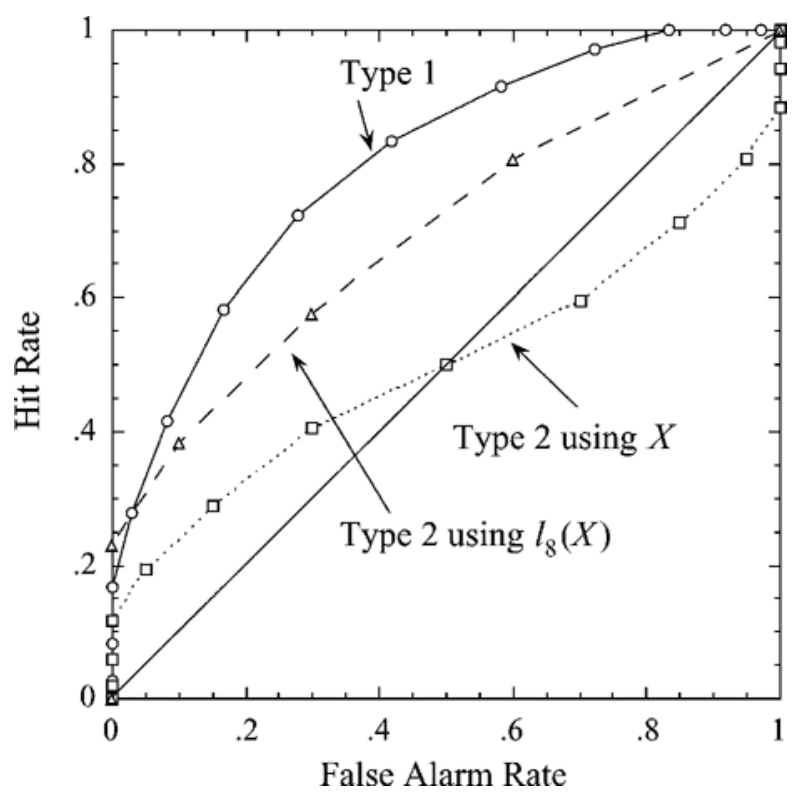

Figure 3. Receiver-operating characteristic curves for a Type 1 task (solid line) and for Type 2 tasks based on $X$ and a Type 1 criterion of $k=8$ (dotted line) and on $l_{8}(X)$ (dashed line).

$\cap C$ ] column is $P_{8}(C)=.72$, the probability of a correct decision given the Type 1 criterion $k=8$.

Note that if a different Type 1 criterion, $k$, had been applied to columns 4 and 5 of Table 1 , some of the joint probabilities that were in the $P_{8}[(X=x) \cap C]$ column would end up in the $P_{8}[(X=x) \cap I]$ column, and some of the joint probabilities that were in the $P_{8}[(X=x) \cap I]$ column would belong in the $P_{8}[(X=x) \cap C]$ column. This would produce not only different values of $P_{k}(I)$ and $P_{k}(C)$, but also different Type 2 probability functions. In general, each distinct Type 1 criterion produces a different

Table 1

Type 1 Probability Functions and Joint Probabilities of Type 1 Events and Values of Evidence

\begin{tabular}{ccccc}
\hline \multirow{2}{*}{$\begin{array}{c}\text { Sum } \\
(x)\end{array}$} & \multicolumn{2}{c}{ Type 1 Functions } & & \multicolumn{2}{c}{ Joint Probabilities } \\
\cline { 2 - 3 } \cline { 5 - 6 }$P(X=x \mid N)$ & $P(X=x \mid S)$ & & $P[(X=x) \cap N]$ & $P[(X=x) \cap S]$ \\
\hline 2 & $1 / 36$ & 0 & $1 / 72$ & 0 \\
3 & $2 / 36$ & 0 & $2 / 72$ & 0 \\
4 & $3 / 36$ & 0 & $3 / 72$ & 0 \\
5 & $4 / 36$ & $1 / 36$ & $4 / 72$ & $1 / 72$ \\
6 & $5 / 36$ & $2 / 36$ & $5 / 72$ & $2 / 72$ \\
7 & $6 / 36$ & $3 / 36$ & $6 / 72$ & $3 / 72$ \\
8 & $5 / 36$ & $4 / 36$ & $5 / 72$ & $4 / 72$ \\
9 & $4 / 36$ & $5 / 36$ & $4 / 72$ & $5 / 72$ \\
10 & $3 / 36$ & $6 / 36$ & $3 / 72$ & $6 / 72$ \\
11 & $2 / 36$ & $5 / 36$ & $2 / 72$ & $5 / 72$ \\
12 & $1 / 36$ & $4 / 36$ & $1 / 72$ & $4 / 72$ \\
13 & 0 & $3 / 36$ & 0 & $3 / 72$ \\
14 & 0 & $2 / 36$ & 0 & $2 / 72$ \\
15 & 0 & $1 / 36$ & 0 & $1 / 72$ \\
Total & 1 & 1 & $P(N)=.5$ & $P(S)=.5$ \\
\hline
\end{tabular}


Table 2

Joint Probabilities of Type 2 Events and Values of Evidence, Type 2 Probability Functions, and Type 2 Likelihood Ratios

\begin{tabular}{rccccc}
\hline$(x)$ & $P_{8}[(X=x) \cap I]$ & $P_{8}[(X=x) \cap C]$ & $P_{8}[(X=x) \mid I]$ & $P_{8}[(X=x) \mid C]$ & $l_{8}(x)$ \\
\hline 2 & 0 & $1 / 72$ & 0 & $1 / 52$ & $\infty$ \\
3 & 0 & $2 / 72$ & 0 & $2 / 52$ & $\infty$ \\
4 & 0 & $3 / 72$ & 0 & $3 / 52$ & $\infty$ \\
5 & $1 / 72$ & $4 / 72$ & $1 / 20$ & $4 / 52$ & $20 / 13$ \\
6 & $2 / 72$ & $5 / 72$ & $2 / 20$ & $5 / 52$ & $25 / 26$ \\
7 & $3 / 72$ & $6 / 72$ & $3 / 20$ & $6 / 52$ & $10 / 13$ \\
8 & $4 / 72$ & $5 / 72$ & $4 / 20$ & $5 / 52$ & $25 / 52$ \\
9 & $4 / 72$ & $5 / 72$ & $4 / 20$ & $5 / 52$ & $25 / 52$ \\
10 & $3 / 72$ & $6 / 72$ & $3 / 20$ & $6 / 52$ & $10 / 13$ \\
11 & $2 / 72$ & $5 / 72$ & $2 / 20$ & $5 / 52$ & $25 / 26$ \\
12 & $1 / 72$ & $4 / 72$ & $1 / 20$ & $4 / 52$ & $20 / 13$ \\
13 & 0 & $3 / 72$ & 0 & $3 / 52$ & $\infty$ \\
14 & 0 & $2 / 72$ & 0 & $2 / 52$ & $\infty$ \\
15 & 0 & $1 / 72$ & 0 & $1 / 52$ & $\infty$ \\
Total & $P_{8}(I)=20 / 72$ & $P_{8}(C)=52 / 72$ & 1 & 1 & \\
\multicolumn{7}{c}{$=$} & $=.72$ & & & \\
\hline
\end{tabular}

pair of Type 2 probability functions and a different Type 2 ROC curve.

Finally, the Type 2 probability distributions are obtained using the definition of conditional probability:

$$
P(A \mid B)=\frac{P(A \cap B)}{P(B)}, \quad P(B) \neq 0 .
$$

For example (from Table 2),

$$
\begin{aligned}
P_{8}(X=10 \mid C) & =\frac{P_{8}[(X=10) \cap C]}{P_{8}(C)} \\
& =\frac{6 / 72}{52 / 72} \\
& =6 / 52 .
\end{aligned}
$$

Similarly, each entry in columns 2 and 3 can be divided by the total of the column in which it appears [i.e., by $P_{8}(C)$ or $\left.P_{8}(I)\right]$, giving the probability distributions conditional on the events $C$ and $I$, shown in columns 4 and 5 of Table 2. These are plotted in Figure 4, and the Type 2 ROC curve for the decision axis, $X$, is shown as the dotted line in Figure 3.

Note that the Type 2 ROC curve lies partly below the positive diagonal, which is the line indicating chance performance. Points lying well below the chance line show discrimination between correct and incorrect decisions that is just as good as that recorded by points well above the chance line, in the sense that the observer can be expected to give, in general, different Type 2 responses on correct and incorrect trials. Points below the chance line indicate, however, that for the criteria that yield these points, the observer will report the majority of correct decisions as incorrect, and vice versa. This is to be expected in the case in which an observer used $X$ as the Type 2 decision axis. For the smallest values of evidence, the observer reports that a noise event occurred but then must give the Type 2 decision that that choice was incorrect for any Type 2 criterion $m$ greater than that value of evidence $x$, because the Type 2 decision rule based on $X$ requires saying "My decision was incorrect" if $x \leq m$. As with Type 1 ROC curves, having parts of the ROC curve fall below the chance line indicates that better decision axes are available (see Egan, 1975, p. 40).

We will now show how to produce Type 2 probability functions for the case in which the likelihood ratio of the Type 2 probability functions, $l_{8}(X)=P_{8}(X=x \mid C) / P_{8}(X=$ $x \mid I)$, is used as the decision axis for the Type 2 task. We do this because predictions for the likelihood ratio observer provide the upper bound on performance for all possible decision axes for the Type 2 task. ${ }^{10}$ We have derived probability functions for $l_{8}(X)$ conditional on $C$ and $I$ from $f_{8}(x \mid C)$ and $f_{8}(x \mid I)$ in the same way one would derive probability functions for $l(X)=P(X \mid S) / P(X \mid N)$ from $f(x \mid S)$ and $f(x \mid N)$. The values of $l_{8}(X)$ are shown in the last column of Table 2 . Some of the evidence values have the same likelihood ratios, so when the likelihood ratio values are rank ordered in Table 3 , all the probabilities for each likelihood ratio are combined within a column. For example, $l_{8}(x)=25 / 26$ appears twice, and its probability conditional on $I$ is the sum of $2 / 20$ and $2 / 20$.

The distributions in Table 3 can be used to produce a Type 2 ROC curve for decision axis $l_{8}(X)$, shown by the dashed line in Figure 3. Note that this curve lies well below

Table 3

Type 2 Probability Functions for Type 2 Likelihood Ratio Observer

\begin{tabular}{ccc}
\hline $\begin{array}{c}\text { Type } 2 \\
\text { Likelihood Ratio } \\
{\left[l_{8}(x)\right]}\end{array}$ & \multicolumn{2}{c}{ Type 2 Probability Functions } \\
\cline { 2 - 3 } & $P_{8}\left[\left(l_{8}(X) \mid I\right]\right.$ & $P_{8}\left[\left(l_{8}(X) \mid C\right]\right.$ \\
\hline $25 / 52$ & $8 / 20$ & $10 / 52$ \\
$10 / 13$ & $6 / 20$ & $12 / 52$ \\
$25 / 26$ & $4 / 20$ & $10 / 52$ \\
$20 / 13$ & $2 / 20$ & $8 / 52$ \\
$\infty$ & 0 & $12 / 52$ \\
Total & 1 & 1 \\
\hline
\end{tabular}




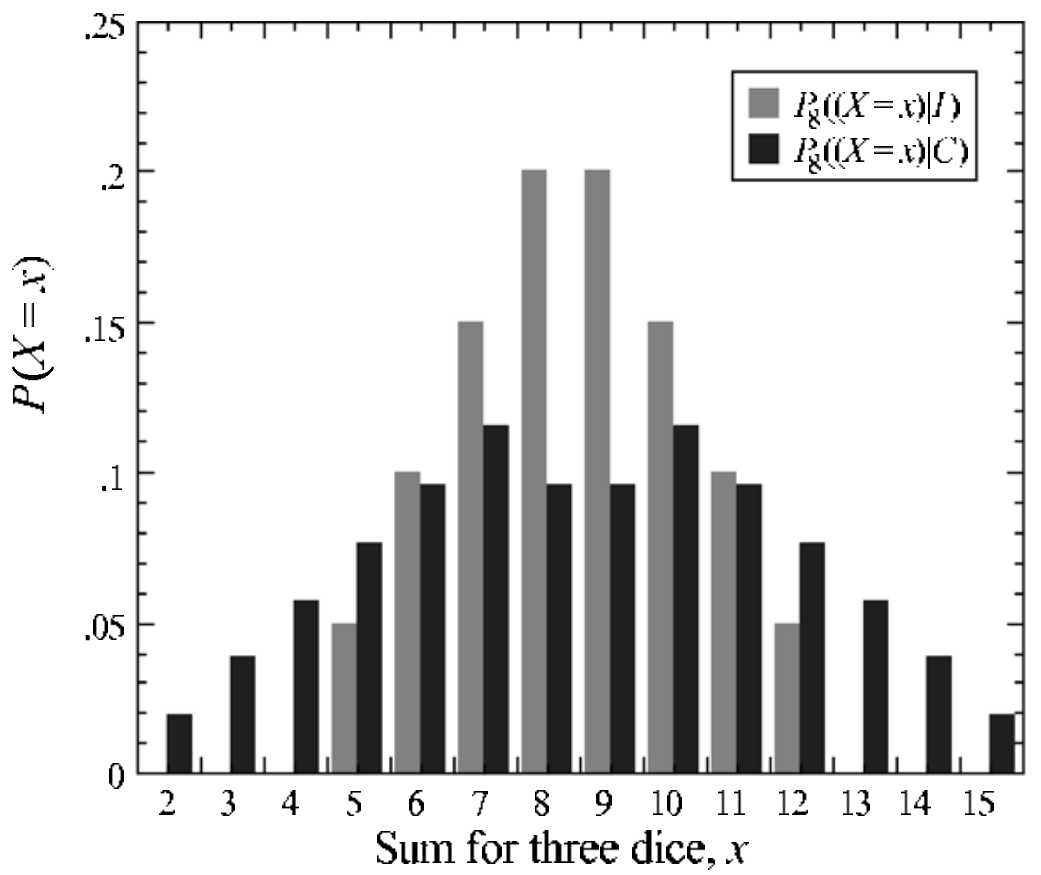

Figure 4. Type 2 mass functions for Type 1 criterion $k=8$.

the Type 1 ROC curve, even though the hypotheticalobserver used the Type 1 criterion maximizing percentage correct in the Type 1 task and the optimal Type 2 decision axis for that case-namely, $l_{8}(X)$. Drawing attention to the difference between these two curves is one of the main purposes of this article, because it is stark evidence that the difference between Type 1 and Type 2 tasks is not merely procedural.

\section{Families of Type 2 Curves}

In TSD, the term family of ROC curves usually refers to the set of curves obtained by varying some parameter in either or both of the underlying probability functions. Each curve may represent a different signal-to-noise ratio, for example. The members of a family of Type 2 ROC curves are all obtained using the same pair of Type 1 functions, but each member of the family is produced by the use of a different Type 1 criterion. Figure 5 shows the family of Type 2 ROC curves for all possible criteria for the Type 1 probability functions in Table 1 . When $k<2$, the observer must say "yes, signal" on every trial and is correct only on $S$ trials. This puts all and only the joint probabilities involving the $S$ event in the column of joint probabilities involving $C$, making the Type 2 functions for $k<2$ equal to the Type 1 functions. The ROC curve for $k<2$ in Figure 5 can be seen to be the same as the Type 1 ROC curve in Figure 3. Also, when Type 2 curves are based on $X$, there is often a sharp change in the slope of the ROC curve at the point where the Type 2 criterion matches the Type 1 criterion. The ROC curve for $k=10$ has been highlighted in Figure 5 to make the distinct knee in the curve readily seen.
A different pair of probability distributions of $l_{k}(x)$ can be derived for each Type 1 criterion, $k$ giving the family of Type 2 curves shown in Figure 6. Like all ROC curves based on a likelihood ratio decision axis, these curves all lie above

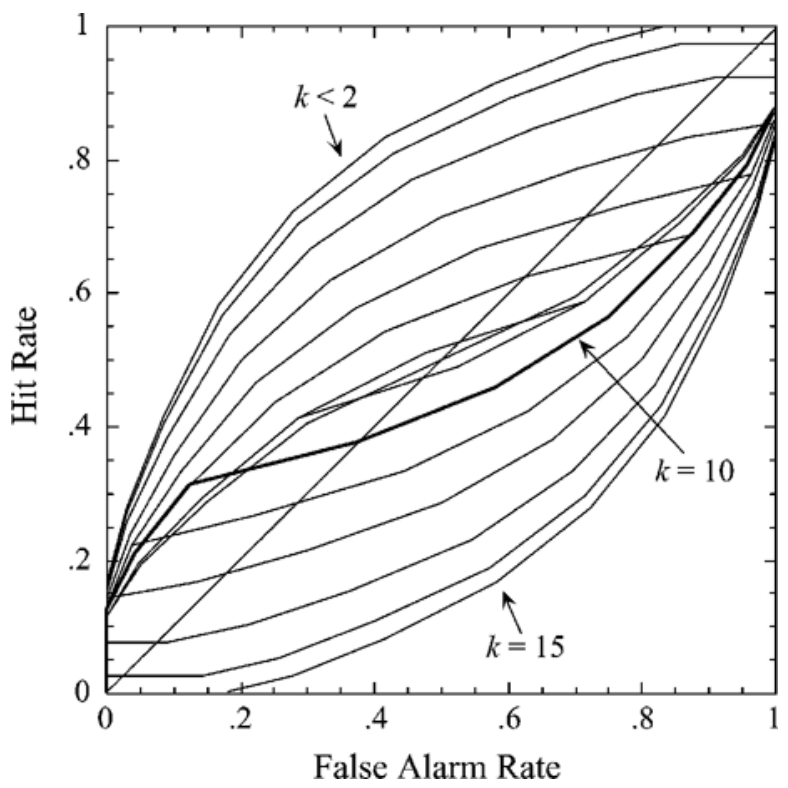

Figure 5. The family of Type 2 receiver operating characteristic (ROC) curves for all possible Type 1 criteria applied to the Type 1 probability distributions shown in Figure 2. The decision axis used for the Type 2 task here is $X$. The Type 2 ROC curve for $k<2$ is the same as the Type 1 ROC curve shown in Figure 3. 


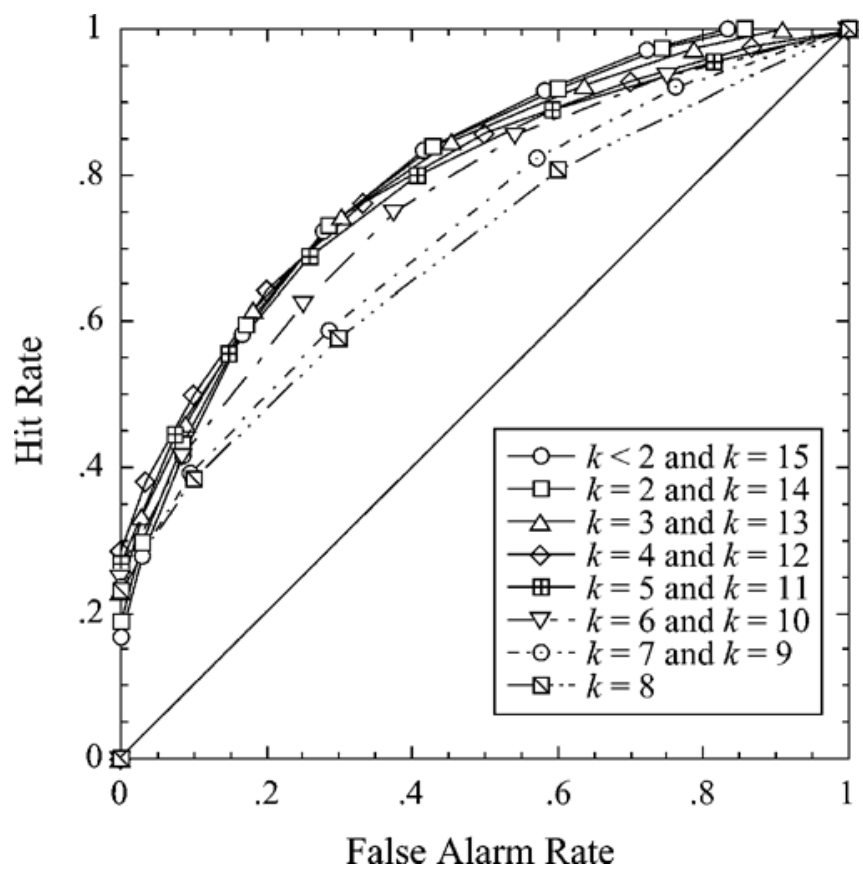

Figure 6. The family of Type 2 receiver operating characteristic curves for all the Type 1 criteria in the dice game, with $l_{k}(X)$ used as the decision axis.

the chance line and a monotonically decreasing slope (Egan, 1975 , chap. 2). Because of the symmetry of the Type 2 distributions in Figure 4, the ROC curves for the Type 1 criteria less than 8 are each identical to one of the ROC curves for the criteria greater than 8 . The Type 2 curve for $k<2$ is the same as the Type 1 curve shown in Figure 3, because $X$ varies monotonically with $l(X)$ in the dice game.

It is interesting to consider how maximum possible performance changes when the Type 1 criterion for the dice game is varied. The first panel in Figure 7 shows the percentage correct for the Type 1 task and the area under the Type 2 ROC curve plotted against the Type 1 criterion. For this task, the Type 1 criterion that produces the highest percentage correct in the Type 1 task produces the poorest performance in the Type 2 task. The areas under all the Type 2 ROC curves are less than or equal to the area under the Type 1 ROC (shown by the heavy dashed line) even though parts of these curves lie above the Type 1 ROC curve

Table 4

General Equations for Type 2 Probability Functions

\begin{tabular}{ccc}
\hline & $x \leq k$ & $x>k$ \\
\hline$f_{k}(x \mid I)$ & $\frac{f(x \mid S) \cdot P(S)}{P_{k}(I)}$ & $\frac{f(x \mid N) \cdot P(N)}{P_{k}(I)}$ \\
$f_{k}(x \mid C)$ & $\frac{f(x \mid N) \cdot P(N)}{P_{k}(C)}$ & $\frac{f(x \mid S) \cdot P(S)}{P_{k}(C)}$ \\
\hline
\end{tabular}

Note- $k$ is the Type 1 criterion on the $X$ axis. (see Figure 6). The maximum percentage correct obtainable in a binary Type 2 task based on $l_{k}(X)$ is the same regardless of Type 1 criterion and is equal to the maximum percentage correct in the Type 1 task (shown by the light dashed line). A Type 2 criterion on the $l_{k}(X)$ axis can always be found that gives this performance for any $k$ (see Corollary 5 below).

If the dice game is changed slightly, so that there are five zeros on the odd die and only one three, then the probability of an $S$ event is reduced to one sixth, and the maximum possible performances change accordingly. This can be seen in the second panel of Figure 7, which shows that the area under some of the Type 2 ROC curves based on $l_{k}(X)$ exceeds the area under the Type 1 ROC curve (marked by the heavy dotted line in Figure 7). Also, there is no longer a simple inverse relationship between percentage correct for the Type 1 task and the area under the Type 2 ROC curve. Such complicated relationships are common in the Type 2 models we have derived.

\section{Derivation of General Equations for Type 2 Functions}

The following derivation results in a set of equations from which the Type 2 probability functions of a decision variable can be obtained using any pair of Type 1 probability functions of that variable and a Type 1 criterion on that decision variable. To keep things as simple as possible, we will refer to the decision variable as $X$, but the decision variable can be any transformation of the evidence 

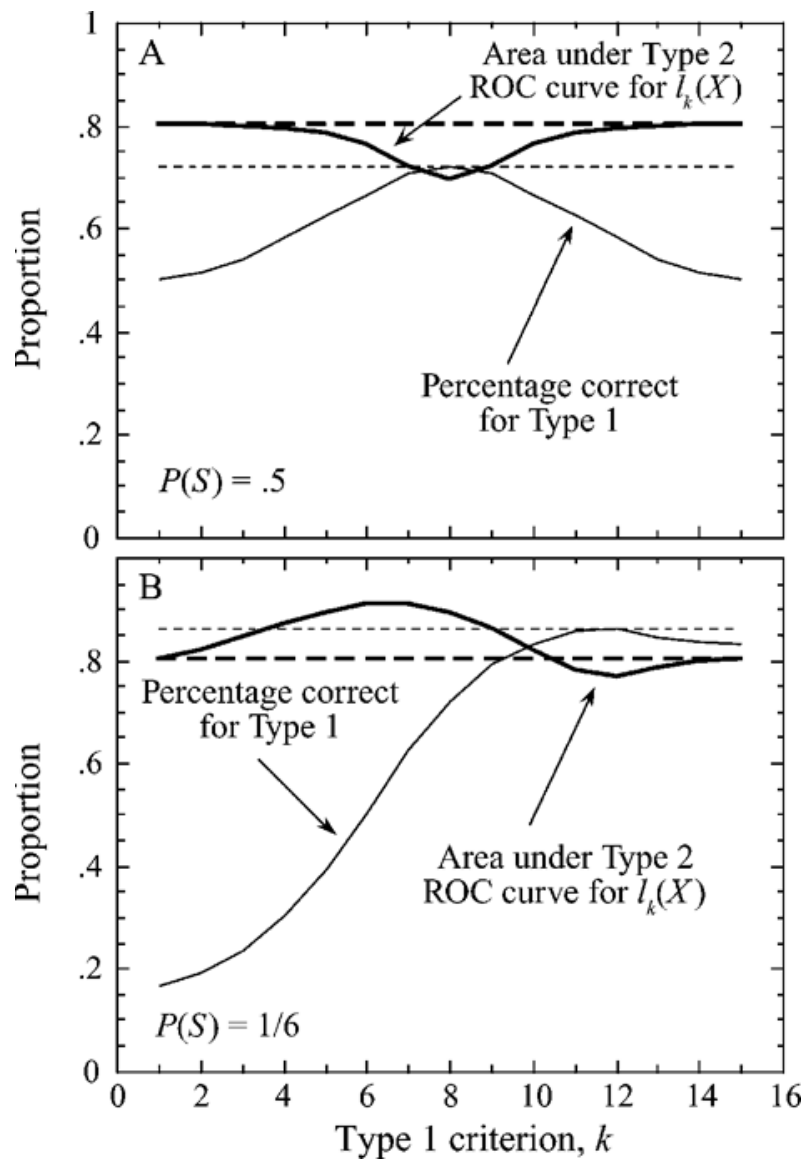

Figure 7. Percentage correct in the Type 1 task (light lines) and the area under the Type 2 curve (heavy lines) for an $l_{k}(X)$ observer for all possible Type 1 criteria in the dice game. (A) $P(S)=.5$. (B) $P(S)=1 / 6$. Horizontal lines show the area under the Type 1 receiver operating characteristic (ROC) curve (heavy line) and the maximum percent correct obtainable in the Type 1 task (light line).

variable, $V=t_{1}(X)$. The Type 2 probability functions of the Type 1 decision variable can then be used to derive probability functions for other decision axes that are transformations of $X$.

The derivation that follows generalizes the method for obtaining Type 2 probability functions from any pair of Type 1 probability functions. The method is embodied in the expressions in Table 4. It is not necessary to follow every step of the derivation in order to be able to apply the equations in Table 4, but following through the derivation will give the reader confidence in Table 4 .

We have illustrated the proof by deriving Type 2 distributions from a pair of normal probability density functions with equal variance. However, the proof applies to both discrete and continuous probability functions of any form. The equations are also general with respect to the Type 1 prior probabilities, although these probabilities are equal $[P(S)=P(N)=.5]$ in the example.
The hit rate for the Type 1 task for any criterion, $k$, is the probability that the value, $x$, of the evidence variable, $X$, exceeds that criterion, given that a signal event, $S$, occurs. That gives

$$
H R_{1}(k)=P(X>k \mid S) .
$$

The hit rate for the Type 2 task for any criterion, $m$, is the probability that the same value of the evidence, $x$, exceeds $m$, given that $k$ was the criterion used in the Type 1 task and given that the observer's Type 1 decision was correct:

$$
H R_{2, k}(m)=P_{k}(X>m \mid C) \text {. }
$$

By the definition of conditional probability,

$$
P_{k}(X>m \mid C)=\frac{P_{k}[(X>m) \cap C]}{P_{k}(C)}, \quad P_{k}(C) \neq 0,
$$

where $P_{k}[(X>m) \cap C]$ is the probability that $X>m$ and the Type 1 decision (using $k$ as the criterion) was correct and $P_{k}(C)$ is the probability of a correct Type 1 decision using $k$ as the criterion.

An equation for $P_{k}(C)$ based on the Type 1 probability functions can be obtained as follows. In the Type 1 task, the observer will state "yes" to all values of $X$ that exceed $k$, so these values of $X$ will result in a correct decision only if they occur during a signal interval. The observer states "no" to values of $X$ less than or equal to $k$, which will be the correct decision only if those values of $X$ are associated with the noise event. Thus,

$P_{k}(\mathrm{C})=P(X>k \mid S) \cdot P(S)+P(X \leq k \mid N) \cdot P(N)$.

The probability of an incorrect decision in the Type 1 task is

$P_{k}(I)=P(X \leq k \mid S) \cdot P(S)+P(X>k \mid N) \cdot P(N)$.

We will now derive an expression, based on the Type 1 probability functions, for the numerator in the right-hand side of Equation 3, $P_{k}[(X>m) \cap C]$. Remember that $m$ is the criterion used in the Type 2 task, and $x$ is the value of $X$ occurring on a particular trial. The observer will say "yes, correct" if $x>m$ and "no, incorrect" if $x \leq m$. If $m$ $>k, x$ can exceed $m$ only if $x$ also exceeds $k$. Therefore, for $m>k$, the only time that $x>m$ and a correct Type 1 decision is made is when $x>m$ for a signal event. The probability of this happening on any trial is $P(X>m \mid S)$. $P(S)$. If $m \leq k$, the condition $x>m$ allows either $x \leq k$ or $x>k$ (see Figure 8). If $x>m$ and $x \leq k$, the Type 1 decision will have been correct only if a noise event occurred. The probability $P(m<X \leq k \mid N)$ is shaded vertically in Figure 8. If $x>m$ and $x>k, x$ must have been associated with a signal event for the Type 1 decision to have been correct. The horizontally shaded area in Figure 8 is $P(X>k \mid S)$. Therefore,

$$
\begin{aligned}
P_{k}[(X>m) \cap C]= & P(m<x \leq k \mid N) \cdot P(N) \\
& +P(X>k \mid S) \cdot P(S), \quad \text { for } m \leq k .
\end{aligned}
$$

Equation 3 becomes [for $P_{k}(C) \neq 0$ ] the following: 


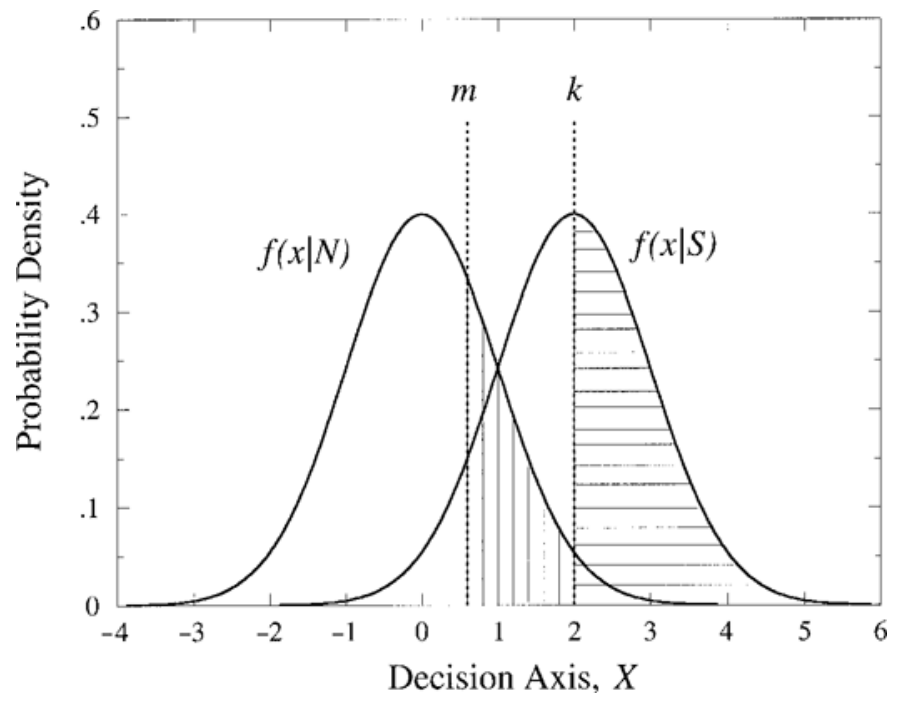

Figure 8. Normal density functions for $N(0,1)$ and $N(2,1)$ showing probabilities contributing to $P_{k}[(X>m) \cap C]$ for the condition $x \leq k$. The vertically shaded area is $P(x<X \leq k \mid N)$, and the horizontally shaded area is $P(X>k \mid S)$.

$$
\begin{aligned}
P_{k}(X>m \mid C)= & \left\{\begin{array}{l}
\frac{P(X \leq k \mid N) \cdot P(N)-P(X \leq m \mid N) \cdot P(N)+P(X>k \mid S) \cdot P(S)}{P_{k}(C)}, \text { for } m \leq k \\
\frac{P(X>m \mid S) \cdot P(S)}{P_{k}(C)}, \text { for } m>k
\end{array}\right. \\
= & \left\{\begin{array}{l}
\frac{P_{k}(C)-P(X \leq m \mid N) \cdot P(N)}{P_{k}(C)}, \text { for } m \leq k \\
\frac{P(X>m \mid S) \cdot P(S)}{P_{k}(C)}, \text { for } m>k
\end{array}\right. \\
& =\left\{\begin{array}{l}
1-\frac{F(m \mid N) \cdot P(N)}{P_{k}(C)}, \text { for } m \leq k \\
\frac{[1-F(m \mid S)] \cdot P(S)}{P_{k}(C)}, \text { for } m>k .
\end{array}\right.
\end{aligned}
$$

By a similar argument, values on the abscissa of the Type 2 ROC curve are given [for $P_{k}(I) \neq 0$ ] by

$$
\mathrm{FAR}_{2, k}(m)= \begin{cases}1-\frac{F(m \mid S) \cdot P(S)}{P_{k}(I)}, & \text { for } m \leq k \\ \frac{[1-F(m \mid N)] \cdot P(N)}{P_{k}(I)}, & \text { for } m>k .\end{cases}
$$

In summary, the equations for points on the Type 2 ROC curve for $X$ as the decision axis, given $k$ as the Type 1 criterion, and presented as functions of the Type 2 criterion, $m$, are shown in Table 5 .

The Type 2 cumulative distribution functions can be obtained as follows: ${ }^{11}$

$$
F_{k}(x \mid C)=P_{k}(X \leq x \mid C)=1-P_{k}(X>x \mid C)
$$$$
=\left\{\begin{array}{l}
1-\left[\frac{F(x \mid N) \cdot P(N)}{P_{k}(C)}\right], \text { for } x \leq k \text { from Equation6a } \\
1-\frac{[1-F(x \mid S)] \cdot P(S)}{P_{k}(C)}, \text { for } x>k, \text { from Equation6b }
\end{array}\right.
$$$$
= \begin{cases}\frac{F(x \mid N) \cdot P(N)}{P_{k}(C)}, \quad \text { for } x \leq k & \\ \frac{P_{k}(C)-P(S)+F(x \mid S) \cdot P(S)}{P_{k}(C)}, & \text { for } x>k,\end{cases}
$$ 
Table 5

Points on the Type 2 ROC Curve

\begin{tabular}{lcc}
\hline & $m \leq k$ & $m>k$ \\
\hline$F A R_{2, k}(m)$ & $1-\frac{F(m \mid S) \cdot P(S)}{P_{k}(I)}$ & $\frac{[1-F(m \mid N)] \cdot P(N)}{P_{k}(I)}$ \\
$H R_{2, k}(m)$ & $1-\frac{F(m \mid N) \cdot P(N)}{P_{k}(C)}$ & $\frac{[1-F(m \mid S)] \cdot P(S)}{P_{k}(C)}$ \\
\hline
\end{tabular}

Note-The Type 1 criterion is $k$ and the Type 2 criterion is $m$, each applied to the decision axis $X$.

Similarly,

$$
F_{k}(x \mid I)=\left\{\begin{array}{l}
\frac{F(x \mid S) \cdot P(S)}{P_{k}(I)}, \text { for } x \leq k \\
\frac{P_{k}(I)-P(N)+F(x \mid N) \cdot P(N)}{P_{k}(I)}, \text { for } x>k .
\end{array}\right.
$$

The probability functions of $X$ conditional on $C$ and on $I$ (which can be discrete or continuous) can be obtained from these cumulative distribution functions by taking the derivative of $F_{k}(x \mid C)$ and $F_{k}(x \mid I)$ in the continuous case and by considering the limits of $F_{k}(x \mid C)$ and $F_{k}(x \mid I)$ from above and below in the discrete case.

For the continuous case,

$$
f_{k}(x \mid C)=\left\{\begin{array}{l}
\frac{f(x \mid N) \cdot P(N)}{P_{k}(C)}, \text { for } x \leq k \\
\frac{f(x \mid S) \cdot P(S)}{P_{k}(C)}, \text { for } x>k,
\end{array}\right.
$$

because $P(S)$ and $P_{k}(C)$ are both constants.

For the discrete case, $f_{k}(x \mid C)$ refers to a probability mass function and is given by the equation at the top of page 15 , where $f_{k}(x \mid N)$ and $f_{k}(x \mid S)$ denote the Type 1 probability mass functions.

Similarly, using Equations 9a and 9b, it can be shown that for both the discrete and the continuous cases,

$$
f_{k}(x \mid I)= \begin{cases}\frac{f(x \mid S) \cdot P(S)}{P_{k}(I)}, & \text { for } x \leq k \\ \frac{f(x \mid N) \cdot P(N)}{P_{k}(I)}, & \text { for } x>k .\end{cases}
$$

In summary, the Type 2 probability functions can be derived from the Type 1 probability functions by using the equations in Table 4 . The area under each of $f_{k}(x \mid C)$ and $f_{k}(x \mid I)$ can be shown to be 1 for both continuous and discrete cases, as is required of a probability function.

Figure 9 illustrates the Type 2 density functions obtained when the equations in Table 4 are applied to the density functions in Figure 8. The Type 1 functions here are $N(0,1), N(2,1)$, and $k=2$. For equal priors, this gives $P_{k=2}(C)=.739$. The Type 2 functions are obtained by scaling the regions of each Type 1 density to the left and right of the criterion by the factors indicated in Table 4 . For example, the region of $f_{k=2}(x \mid C)$ to the right of 2 is the region of the $N(2,1)$ function to the right of 2 , scaled by $.5 / .739$, or .677 . In general, Type 2 probability functions based on continuous Type 1 probability functions of $X$ will be discontinuous, like these, unless $l(k)=P(N) / P(S)$.

Figure 10 shows a family of Type 2 curves using $X$ as the decision axis obtained from the Type 1 densities $N(0,1)$ and $N(2,1)$, using criteria $k=-4,-1,0,1,2,3$, and 6 . Note that the complete family has an infinite number of ROC curves, because any real number can serve as the Type 1 criterion on the decision axis in this example.

Finally, a family of Type 2 curves for $l_{k}(X)$ as decision axis is shown in Figure 11. The curves for $k=0,-1$, and -4 are the same as the curves for $k=2,3$, and 6 and so are not shown. The Type 2 ROC curve for the extreme criteria, -4 and 6 , is virtually the same as the Type 1 curve and is invisible in the figure. Note that the ROC curves for $k=1,2$, and 3 are not symmetrical about the negative diagonal: The Type 2 probability functions underlying these ROC curves are not Gaussian with equal variance. (Thus, even if some Type 1 task meets the criteria allowing the use of $d^{\prime}$, this does not mean it is appropriate to use $d^{\prime}$ as a measure of Type 2 performance.) The derivation of the Type 2 probability functions of $l_{k}(X)$ is similar to that given in Appendix A but is a little more complicated when the Type 1 criterion is not halfway between the peaks of the two Type 1 probability functions and is longer than can be presented here.

\section{Combining Different Decision Axes \\ for the Type 1 and Type 2 Tasks}

A key observation to be made about models for Type 2 performance is that the decision axes for the Type 1 and the Type 2 tasks may be different and should be stated clearly in any model. We introduce here the notation $1: t_{1}(X) ; 2: t_{2}(X)$ to indicate that the transformations $t_{1}(X)$ and $t_{2}(X)$ have been used as the decision axes for Type 1 and Type 2 decisions. For example, one possible model for performance in the dice game with unequal priors is that the observer uses $X$ as the Type 1 decision axis, adopts criterion $k=10$, and uses distance from the Type 1 criterion as the Type 2 decision axis. The notation for this is $1: X ; 2:|X-10|$. The model for an observer using $k=10$ and the likelihood ratio of the Type 2 functions as the Type 2 decision axis is referred to as $1: X ; 2: l_{10}(X)$. 


$$
\begin{aligned}
& f_{k}(x \mid C)= \lim _{h \rightarrow 0} F_{k}(x+h \mid C)-\lim _{h \rightarrow 0} F_{k}(x-h \mid C) \\
&=\left\{\begin{array}{l}
\lim _{h \rightarrow 0} \frac{F(x+h \mid N) \cdot P(N)}{P_{k}(C)}-\lim _{h \rightarrow 0} \frac{F(x-h \mid N) \cdot P(N)}{P_{k}(C)}, \text { for } x \leq k \text { from Equation } 8 \mathrm{a} \\
-\lim _{h \rightarrow 0}\left[\frac{P_{k}(C)-P(S)}{P_{k}(C)}+\frac{F(x+h \mid S) \cdot P(S)}{P_{k}(C)}+\frac{F(x-h \mid S) \cdot P(S)}{P_{k}(C)}\right], \text { for } x>k, \text { from Equation } 8 \mathrm{~b}
\end{array}\right. \\
&=\left\{\begin{array}{l}
\frac{P(N)}{P_{k}(C)}\left[\lim _{h \rightarrow 0} F(x+h \mid N)-\lim _{h \rightarrow 0} F(x-h \mid N)\right], \text { for } x \leq k \\
\frac{P(S)}{P_{k}(C)}\left[\lim _{h \rightarrow 0} F(x+h \mid S)-\lim _{h \rightarrow 0} F(x-h \mid S)\right], \text { for } x>k
\end{array}\right. \\
&=\left\{\begin{array}{l}
\frac{f(x \mid N) \cdot P(N)}{P_{k}(C)}, \text { for } x \leq k \\
\frac{f(x \mid S) \cdot P(S)}{P_{k}(C)}, \text { for } x>k .
\end{array}\right.
\end{aligned}
$$

If the Type 1 decision axis is not $X$, it is likely that the Type 2 decision axis will be a transformation of the Type 1 decision axis, and this must be made apparent in the notation. Consider the case of deriving Type 2 performance of an ideal observer for a Type 1 task based on Gaussian probability density functions with different means and unequal variances (one of several possible models of mem- ory tasks in which an observer must decide whether a test word belongs to a previously learned list or is new). Because $X$ and $l(X)$ are not monotonic in this case, a model of optimal Type 1 performance uses $l(X)$ as the Type 1 decision axis. A Type 1 criterion, $\beta$, on $l(X)$ is assumed on the basis of what is known about $P(S)$ and the costs and rewards of hits, misses, false alarms, and correct rejec-

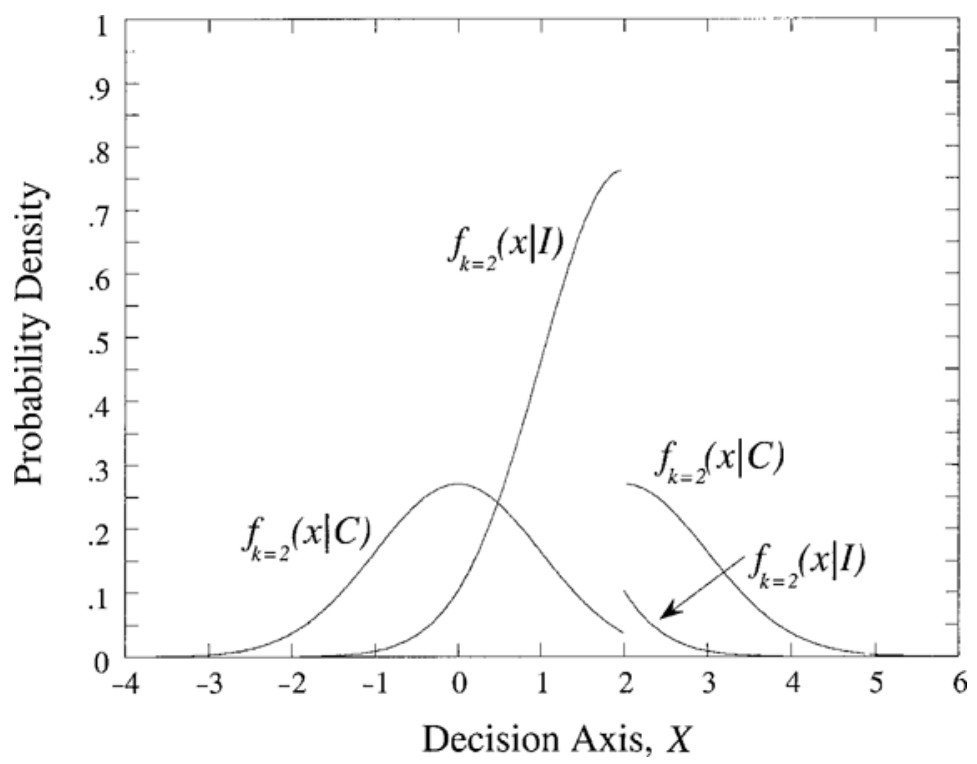

Figure 9. Type 2 probability density functions based on $N(0,1)$ and $N(2,1)$ Type 1 probability density functions with a Type 1 criterion $k=2$. 


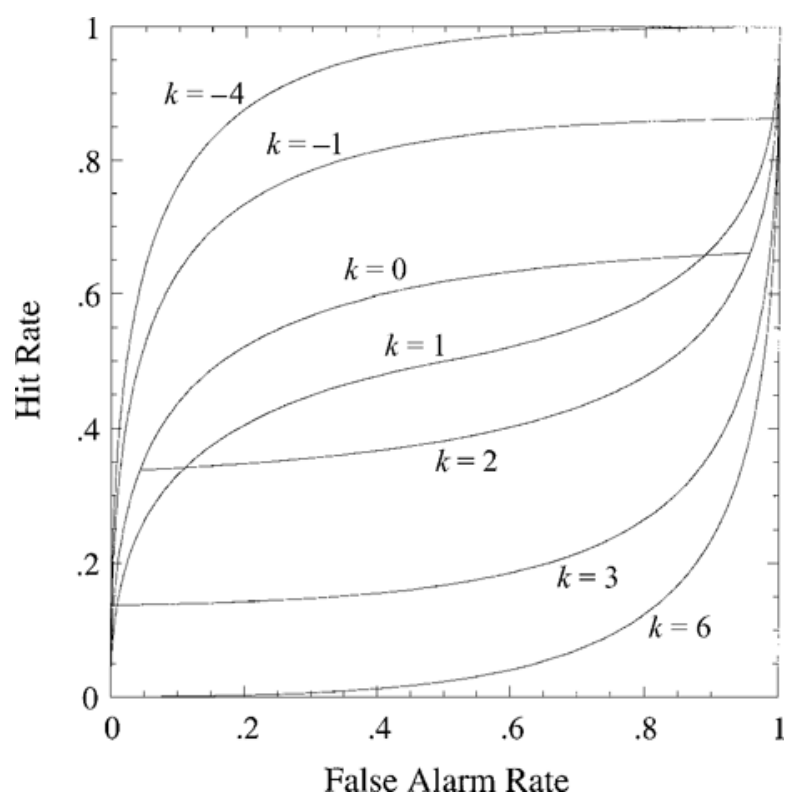

Figure 10. Family of Type 2 receiver operating characteristic curves based on $N(0,1)$ and $N(2,1)$ Type 1 probability density functions for criteria $k=-4,-1,0,1,2,3$, and 6 . The decision axis is $X$ for both Type 1 and Type 2 decisions.

tions. Distributions of $l(X)$ conditional on $S$ and $N$ are derived. The equations in Table 4 are applied to these Type 1 functions, $g[l(x) \mid S]$ and $g[l(x) \mid N]\{$ with $g[l(x) \mid S]$ and $g[l(x) \mid N]$ replacing $f(x \mid S)$ and $f(x \mid N)$ and $\beta$ taking the role of $k\}$, to produce Type 2 functions $g_{\beta}[l(x) \mid C]$ and $g_{\beta}[l(x) \mid I]$. Note that because $l(X)$ is the likelihood ratio of the Type 1 distributions, not of the Type 2 distributions, the theoretical Type 2 ROC curves based on $g_{\beta}[l(x) \mid C]$ and $g_{\beta}[l(x) \mid I]$ could include regions lying below the chance line. It is therefore necessary to take their likelihood ratio, $l_{\beta}[l(X)]=g_{\beta}[l(x) \mid C] / g_{\beta}[l(x) \mid I]$, as the decision axis in the Type 2 task. A transformation of variable would then give the functions underlying the Type 2 ROC curve, $h_{\beta}\left\{l_{\beta}[x] \mid C\right\}$ and $h\left\{l_{\beta}[x] \mid I\right\}$. This task is called $1: l(X)$; $2: l_{\beta}[l(X)]$.

\section{Type 2 Models for Two-Interval Tasks}

We have presented a method of obtaining Type 2 probability functions for tasks in which the Type 1 functions underlying a yes-no task are known. However, theoretical ROC curves can also be derived for two-interval ${ }^{12}$ tasks. For example, the equations in Table 4 can be applied to the probability functions of the variable $W=X_{1}-X_{2}$ conditional on the events " $S$ in interval 1 " and " $S$ in interval 2 ," where $X_{1}$ and $X_{2}$ are the evidence in the first and the second intervals, respectively, to give the Type 2 probability functions of $W .{ }^{13}$ The two-interval task is often described as criterion free, but the common decision rule "Say $S$ was in Interval 1 if $x_{1}>x_{2}$ " is equivalent to "Say $S$ was Interval 1 if $w>0$," so a criterion of zero on $W$ is implied (see also Luce, 1997). A Type 1 ROC curve for the two-interval task can be produced by plotting hit rates and false alarm rates for all possible criteria on $W$, and a family of Type 2 ROC curves, one for each criterion on $W$, can also be derived.

\section{Corollaries to the General Equations}

From the equations in Table 4, the following properties of Type 2 probability functions, ROC curves, and performance measures can be derived. Formal proofs of these corollaries are given in Appendix B.

\section{Corollary 1: Type 2 ROC Curves for Extreme Type 1 Criteria}

The 1:X; 2:X ROC curve obtained using the lowest possible Type 1 criterion (i.e., always saying "yes" in the Type 1 task) is the Type 1 ROC curve; the 1:X; 2:X ROC curve obtained using the highest possible criterion in the Type 1 task (i.e., always say "no") is the reflection of the Type 1 ROC curve in the chance line. The $1: X ; 2: l_{k}(X)$ ROC curve obtained using the highest possible criterion in the Type 1 task is the reflection in the negative diagonal of the 1:X; $2: l_{k}(X)$ ROC curve for the lowest possible criterion.

Because the $1: X ; 2: 1_{-8}(X)$ and $1: X ; 2: 1_{8}(X)$ ROC curves are reflections of each other in the line joining $(0,1)$ and $(1,0)$ in the ROC space, the areas under these two curves are equal. Also, if $X$ and $l(X)$ vary monotonically, this will be the same as the area under the Type 1 curve. This means that if an observer always gives the same answer in the Type 1 task, the subsequent Type 2 task is exactly as difficult as the original Type 1 task (where difficulty is measured by the maximum area under the ROC curve that could be achieved by an ideal observer).

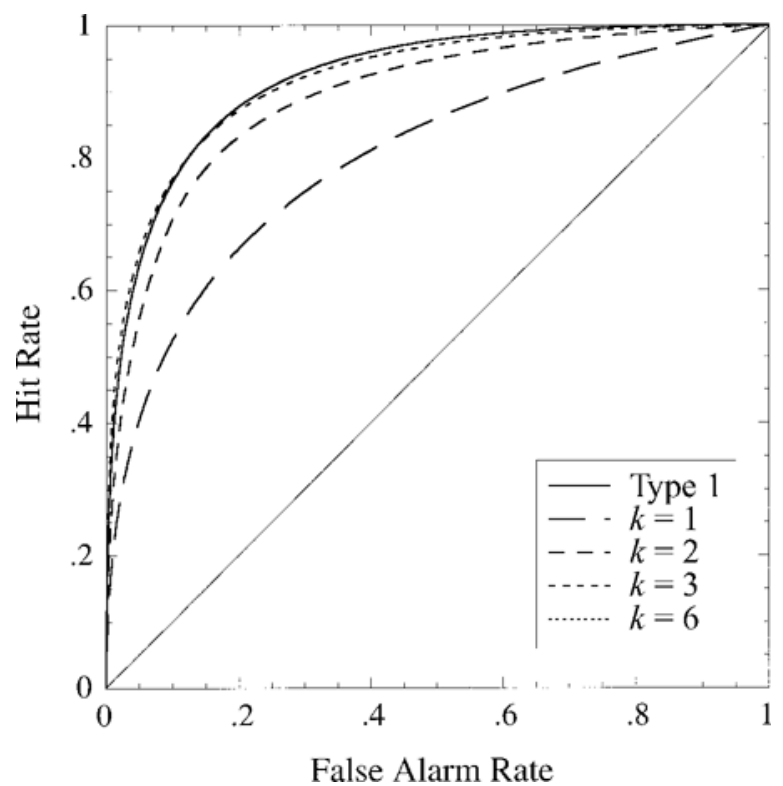

Figure 11. Family of Type 2 receiver operating characteristic (ROC) curves based on $N(0,1)$ and $N(2,1)$ Type 1 probability density functions for Type 1 criteria $k=1,2,3$, and 6 on decision axis $X$; the Type 2 decision axis is $l_{k}(X)$. The Type 2 ROC curve for $k=6$ is covered by the Type 1 ROC curve (solid line). 


\section{COROLLARY 2: Likelihood Ratio for Type 2 Probability Functions}

If $l(X)$ is monotonic with $X$ for $f(x / S)$ and $f(x / N), l_{k}(X)$ is proportional to $1 / l(x)$ for $x \leq k$ and to $l(x)$ for $x>k$. Hence, $l_{k}(x)$ will be monotonic with $X$ only if $k$ is the lowest possible criterion.

This formalizes a point we have made using examplesnamely, that even if $X$ is monotonic with $l(X), X$ will not usually be the optimal Type 2 decision axis. This is not to say that one must use $l_{k}(X)$ as the Type 2 decision axis but only that the use of $X$ might lead to a predicted ROC curve with parts lying below the chance line.

\section{Corollary 3: Type 1 Curve as an Upper Bound for Type 2 ROC Curves}

If $l(X)$ is strictly monotonicallyincreasing with $X$ and $P(S)$ and $P(N)$ are equal, the Type 1 ROC curve puts an upper bound on all the Type 2 ROC curves for which $X$ is the decision axis. The whole Type 2 family of 1:X; 2:X ROC curves lies between the Type 1 curve and its reflection in the chance line.

The fact that the Type 2 curves based on $X$ are all closer to the chance line than the Type 1 ROC curve raises the possibility that the Type 1 curve based on $l(X)$ puts an upper bound on the Type 2 family based on $l_{k}(X)$ for even priors and $l(X)$ monotonic with $X$. We have been unable to prove this so far, but we have not discovered any counterexample either. Note, however, that Figure 7B illustrates that this boundary condition does not hold if the priors are uneven.

\section{Corollary 4: Distance From Type 1 Criterion as Type 2 Decision Axis}

The Type 2 probability functions of the decision variable $W=|X-k|$ are

$$
h(w \mid C)=\frac{f(k-w \mid N) P(N)+f(k+w \mid S) P(S)}{P_{k}(C)}, w \geq 0
$$

and

$h(w \mid I)=\frac{f(k-w \mid S) P(S)+f(k+w \mid N) P(N)}{P_{k}(I)}, w \geq 0$.

Those who have attempted to model how Type 2 ratings are generated using TSD have usually described the ratings as increasing with the absolute distance of the evidence, $x$, or its likelihood ratio, $l(x)$, from the Type 1 criterion on $X$ or $l(X)$, respectively (Baranski \& Petrusic, 1998; Clarke et al., 1959; Van Zandt, 2000). We showed, in Corollary 1 , that if $l(X)$ is monotonic with $X$, the Type 2 likelihood ratio increases as the distance between $X$ and the Type 1 criterion, $k$, increases. However, in Appendix A we show that this relationship does not make $|X-k|$ and Type 2 likelihood ratio equivalent decision axes. Even in the case in which values of $X$ that are the same distance above and below $k$ have the same Type 2 likelihood ratio, the Type 2 probability functions of these decision variables are different.

\section{Corollary 5: Maximum Percentage Correct for the $1: X ; 2: l_{k}(X)$ Task}

The maximum probability of a correct decision attainable in the $1: X ; 2: l_{k}(X)$ task is equal to the maximum probability of a correct decision in the Type 1 task using $l(X)$ as the decision axis.

This is an important result because if the performance measure of interest is simply the proportion of binary Type 2 decisions answered correctly, it shows that, in theory, one can always do as well in the Type 2 task as in the Type 1 task. However, it will usually be the case that the Type 1 task and the Type 2 task will have different prior probabilities - that is, $P_{k}(C) \neq P(S)$. If $P_{k}(C)$ is high, it may be that percentage correct in the Type 2 task is maximized by the observer's always responding "correct." This will make the percentage correct the same for the Type 1 and the Type 2 tasks but will give a point lying on the chance line in the ROC space [the point $(1,1)$ ], revealing no discrimination between correct and incorrect, as will always be the case in any task in which the observer's response is chosen before the evidence is presented. In general, the choice of Type 1 criterion will depend on which Type 2 performance measure the observer wishes to maximize.

\section{Corollary 6: Different Functions with the Same Type 2 ROC Curves}

If two pairs of probability functions give the same Type 1 ROC curve, two criteria (one applied to each pair) that generate a common point on that ROC curve will each generate the same Type 2 ROC curve when applied to their respective probability functions.

An important consequence of Corollary 6 is that to generate a set of predicted Type 2 ROC curves associated with a Type 2 task following a particular Type 1 task, there is no need to know the probability functions underlying the Type 1 task. All that is needed is any pair of probability functions that give a Type 1 curve identical to the obtained curve. Assume that the probability functions $f(x \mid S)$ and $f(x \mid N)$ underlie the Type 1 task (these are the unknown ones) and that Type 1 criterion $k_{1}$ gives a particular point on the observed Type 1 curve. Let $q(x \mid S)$ and $q(x \mid N)$ be another pair of probability functions that give the same Type 1 ROC curve as $f(x \mid S)$ and $f(x \mid N)$. There will exist some Type 1 criterion, $k_{2}$, that when applied to $q(x \mid S)$ and $q(x \mid N)$, will give that same point on the Type 1 curve. The pair of Type 2 probability functions produced by using $k_{2}$, $q(x \mid S)$, and $q(x \mid N)$ in the equations in Table 4 will generate the same Type 2 ROC curve as the pair of Type 2 probability functions produced by using $k_{1}, f(x \mid S)$, and $f(x \mid N)$ in the equations in Table 4, providing a prediction 
for Type 2 performance, given the observed Type 1 performance.

Note that to predict performance for a set of Type 2 trials using some set of stimuli, a separate set of trials using the same stimulus set and a Type 1 rating task can be used to generate a pair of probability functions that can serve as $q(x \mid S)$ and $q(x \mid N)$. The function $q(x \mid N)$ can be obtained by taking the number of times each rating was used on $N$ trials and dividing that number by the total number of $N$ trials. Function $q(x \mid S)$ is given by the number of times each rating was used on $S$ trials and dividing by the total number of $S$ trials. This gives two discrete mass functions over a random variable defined by the rating values used. (It does not matter what the actual rating categories are, only that they are ordered.) Because these rating tallies were used to generate the Type 1 ROC curve in the first place, it is known that they give the same ROC curve as the underlying, unknown Type 1 functions, $f(x \mid S)$ and $f(x \mid N)$. By Corollary 6, this qualifies $q(x \mid S)$ and $q(x \mid N)$ as having the same family of Type 2 ROC curves as $f(x \mid S)$ and $f(x \mid N)$.

Although it is a valid method in principle, using $q(x \mid S)$ and $q(x \mid N)$ to predict Type 2 performance is an art yet to be fully developed; a number of factors must be considered. The Type 1 ratings will have been affected by both common and unique noise ${ }^{14}$ and by the observer's ability (or inability) to maintain a consistent set of Type 1 criteria and so might produce distributions $q(x \mid S)$ and $q(x \mid N)$ that are less discriminable than $f(x \mid S)$ and $f(x \mid N)$. Also, it will be necessary to assume a Type 1 criterion on the set of rating categories, which make up a Type 1 decision axis, in order to use the equations in Table 4 to generate Type 2 probability functions. A Type 2 decision axis must also be chosen, which is likely to involve a transformation of the original rating category decision axis. However, in many cases, Type 1 distributions of evidence are not known, and these functions based on Type 1 ratings are all that is available as a means to make predictions.

Note that Type 2 ROC curves predicted in this way should not be considered empirical Type 2 curves, despite being based on empirical data, since the observer has not made any Type 2 decisions to produce them. They are theoretical predictions of performance observers might exhibit if they were to make Type 2 decisions constrained by the assumptions made to generate the Type 2 ROC curve. The fact that there are many choices of Type 2 decision axis is a clear reminder that any such curve is only a prediction, and not an empirical Type 2 curve.

\section{Discussion}

We have presented a general method of generating models of Type 2 performance based on the assumption that on any trial an observer uses the same piece of evidence (albeit transformed somehow) to make both the Type 1 and the Type 2 decisions. Models are produced by assuming Type 1 probability functions of the evidence and a Type 1 decision axis, deriving the transformed Type 1 functions for the Type 1 decision variable if it is not $X$, and applying the equations in Table 4 to generate Type 2 probability functions. A Type 2 decision axis is chosen, and Type 2 probability functions are calculated for the new decision variable. An important revelation of the theory is that Type 2 performance can be quite different from Type 1 performance and is highly dependent on the Type 1 criterion. The relationship between Type 1 and Type 2 discriminations depends on the performance measure chosen, the decision axes chosen for each of the two tasks, the Type 1 criterion used, the shape of the distributions underlying the Type 1 decision, and the prior probabilities of the Type 1 events.

We have derived a number of corollaries from the general equations that have important implications. In particular, we have shown why Type 2 curves based on $X$ as decision axis are likely to drop below the chance line. This indicates that models for empirical Type 2 curves that do not drop below the chance line will require some transformation of $X$ as the Type 2 decision axis. We have shown how the Type 1 ROC curve and its reflection in the chance line puts a bound on the family of Type 2 curves under some conditions and have described how Type 2 curves may be predicted from Type 1 ratings (Corollary 6). We have given examples of how to calculate a transformation of variable for a discrete (the dice game) and a continuous (the model of Clarke et al., 1959) case.

We now return to two interesting issues raised in the introduction - namely, whether it can always be assumed that both the Type 1 and the Type 2 decisions are based on the same evidence and, when it can, what can be said about the relationship between Type 1 and Type 2 confidence ratings.

We raised the question of whether Type 1 and Type 2 decisions are always based on the same value of $X$ in the context of studies of perception without awareness. Kolb and Braun (1995) found excellent Type 1 discrimination accompanied by remarkably poor Type 2 performance. Although we criticized the general method of comparing Type 1 and Type 2 performance to reveal perception without awareness, it is hard to come up with a model that would predict such a large difference between Type 1 and Type 2 performance; it is likely that Type 2 ROC curves predicted from the Type 1 ratings in their study would be higher than the empirical Type 2 ROC curves. Kunimoto et al. (2001), alerted to the problem of directly comparing Type 1 and Type 2 performance, used a model of Type 2 binary confidence ratings based on distance from the intersection of two equal variance Gaussian density functions and $d^{\prime}$ measures to show that Type 1 and Type 2 performance should fall to chance at the same signal-to-noise ratio. A calculation based on our model and the Type 2 likelihood ratio as the Type 2 decision axis gives the same theoretical result. This suggests that in their study, significant Type 1 detection in the presence of a Type $2 d^{\prime}$ of zero was not produced by Type 1 and 2 decisions based on the same source of evidence. It appears that the observer is able to access information when making the Type 1 judgment that cannot be accessed at the time of the Type 2 judgment. This is a very curious result, given that the ob- 
servers in these studies could have done well in the Type 2 task by taking their Type 1 ratings and transforming them into responses appropriate to the Type 2 task. This suggests that the form of the instruction is very important to the observer and that being asked to discriminate and to give a commentary on one's performance causes one to rely on different sources of information. Busey, Tunnicliff, Loftus, and Loftus (2000) influenced Type 2 confidence independently of accuracy in Type 1 decisions by using cues that their observers imagined would improve or degrade their performance. Their data were collected using Type 1 decisions followed by Type 2 ratings, but their measures were of calibration, because they were looking for signs of under- or overconfidence. It would be interesting to apply Type 2 ROC analysis to their data to determine whether the effects of their manipulations were on the Type 2 criterion or on Type 2 discriminability, because only the latter would suggest the use of a different value of evidence for the two decisions.

Imagine a situation in which it is more likely that the observer is using the same evidence for both decisions, such as a memory experiment in which the observer sees a test word and must say whether it belongs to a previously learned list. Consider two tasks: a Type 1 rating task in which the observer rates confidence that the presented word is old and a Type 2 task in which a yes-no Type 1 decision between old and new is followed by a Type 2 confidence rating. What is the difference between these two kinds of confidence ratings, apart from the obvious one that high confidence that the $N$ event occurred (i.e., that the word is new) is indicated by a very low rating in the first case and a very high rating in the second? We have shown that the mathematical constraints on the task of discriminating $S$ from $N$ are different from the constraints on the task of discriminating $C$ from $I$. But one could argue that the difference between Type 1 and Type 2 performance is due just to the difference in the form of the task, analogous to the theoretical difference (a factor of $\sqrt{2}$ ) between the $d^{\prime}$ measures for yes-no and two-interval tasks based on the same pair of Gaussian probability functions. It is tempting to think that the information carried in a binary decision followed by a 4-point Type 2 rating is exactly the same as that carried by an 8-point Type 1 rating. The fact that the theoretical Type 2 distributions are predictable from the theoretical Type 1 distributions (given knowledge of the prior probabilities and the Type 1 criterion) seems to give credence to this view.

The answer to this question is crucial, because it affects the status of what we have called pseudo-Type 1 ratings, obtained by taking a binary choice between $S$ and $N$ followed by a rating on a 4-point scale and expanding these to an 8-point pseudo-Type 1 scale. Macmillan and Creelman (1991) presented pseudo-Type 1 ratings as equivalent to Type 1 ratings and even used a study based on pseudoType 1 ratings to illustrate their chapter on (Type 1) rating experiments (Reber, Kassin, Lewis, \& Cantor, 1980).

We caution against the use of pseudo-Type 1 ratings, because (1) they rely on an untested Type 2 model, (2) they are only predictions of Type 1 ratings, not empirical Type 1 ratings, and (3) there are factors affecting the Type 2 rating decision that would not have affected the Type 1 rating decision the pseudo-Type 1 ratings are meant to mimic.

In generating pseudo-Type 1 ratings from Type 2 ratings, using the algorithm described earlier, the experimenter assumes that the observer is able to lay down $n-1$ criteria on the $n$-point Type 2 decision axis in such a way that when the Type 2 decision axis is unfolded into the pseudo-Type 1 decision axis with $2 n-1$ criteria (the extra criterion being the fold point), the criteria on each side of the fold point will match, or transform to, the criteria the observer would have used on the Type 1 decision axis if he or she had been asked for Type 1 ratings on a $2 n-1$-point scale. It is not easy to come up with realistic models that have this property. Consider the textbook case of Type 1 distributions that are Gaussian with equal variance, a Type 1 criterion applied at the place where the distributions cross, $k$, and Type 2 ratings based on $1: X ; 2:|X-k|$. Here, pseudo- Type 1 ratings generated from the Type 2 ratings can match Type 1 ratings taken using $X$ as the decision axis if the Type 1 rating criteria are symmetrically laid out on either side of $k$. But in any task for which $X$ is not likely to be the Type 1 decision axis or the Type 1 probability functions are not symmetric about the likely binary Type 1 criterion on whatever Type 1 decision axis is used, models that generate Type 1 ROC curves that match pseudo-Type 1 ROC curves get very complicated. Also, to our knowledge, no one has compared Type 1 performance derived from pseudo-Type 1 ratings and actual Type 1 ratings to see whether they do match.

Even if ROC curves generated from Type 1 ratings were found to match ROC curves derived from pseudo-Type 1 ROC curves and someone proposed a Type 2 model that gave a reasonable account of observers' behavior in the tasks used, all that can be said is that good predictions of the Type 1 ratings could be made from the Type 2 ratings under the assumptions of that model. Theoretical assumptions about distributions and decision axes must be made when producing models for data, but it is not a good practice to manipulate data based on assumptions and report these as raw data.

The question of how confidence ratings are generated has attracted many researchers, and there now exists a wealth of literature on calibration and on the relations among accuracy, confidence, and speed of response (see Link, 1992). Because a Type 1 decision followed by a Type 2 rating gives separate measures of accuracy and confidence, the Type 2 task is the standard methodology in this literature (Baranski \& Petrusic, 1998). One line of inquiry of particular interest here is to find out when the confidence ratings are generated. In their model of confidence, Gigerenzer, Hoffrage, and Kleinbölting (1991) assumed that the Type 1 judgment and the (Type 2) confidence assessment are produced at the same time. If this is true, one must consider the possibility that a confidence assessment not reported until the Type 2 decision interval may have degraded, due to the time elapsed between the two decision intervals or to interference from organizing the Type 1 response. One 
might then expect pseudo-Type 1 ratings created from these world-weary Type 2 ratings to carry different information from fresh Type 1 ratings. Baranski and Petrusic suggested that the confidence rating may be decided on during the Type 1 task if the observer is under pressure to get the Type 1 decision correct, and not until afterward if the experimenter stresses making the Type 1 answer quickly. In the latter case, it is difficult to guess how the pseudoType 1 ratings would compare with real Type 1 ratings using the same evidence distributions.

We conclude, therefore, that it is not a good idea to present pseudo-Type 1 ratings as empirical Type 1 ratings, because the theories that predict that Type 1 ROC curves and pseudo-Type 1 ROC curves will be the same will not always hold and, even when they hold, it is unwise to report a prediction as a result. It is an unavoidable aspect of psychological experiments that no matter how well instructed, it is the observer who determines how the task is done. In the perception without awareness experiments, we see that the observer may go so far as to put the response under the control of different processing on the bases of the structure of the tasks and the form of the instruction. If Type 1 ratings are required, experimenters should request them. Finally, if the Type 1 task is of the form that cannot be cast as a rating in the confidence in one of two events (e.g., in the word identification task used by Pollack \& Decker, 1958), the ROC curve generated from the corresponding pseudoType 1 ratings is likely to have no sensible meaning.

\section{Summary and Conclusions}

We have presented a theory for the task of identifying one's own correct decisions in the context of the TSD. The theory applies to a Type 2 binary or rating task following a binary Type 1 decision but will provide the basis for theories of other, more complicated Type 2 tasks. The theory predicts that, in general, the ability to discriminate signal from noise is not equivalent to the ability to discriminate correct from incorrect decisions, even if both decisions are based on the same evidence.

The theory developed here can be used in any situation involving a Type 1 yes-no task followed by a Type 2 binary or rating decision; ${ }^{15}$ studies of sensory discrimination, memory, vigilance, and clinical diagnosis are just some examples. The reason for using a Type 2 analysis will vary accordingly. For example, Podd (1975) was interested in whether giving an observer knowledge of results would effect a change in Type 2 decision axis from evidence, $X$, to likelihood ratio. Since the theoretical ROC curves for the Type 2 task often differ quite markedly for the different decision axes, comparing Type 2 curves from each experimental condition can be a more sensitive test of change in decision axis than comparing Type 1 curves. In another kind of experiment, the ability of observers to assess their own decisions might be under investigation for its own sake, as in the studies of memory and of perception without awareness described earlier.

Empirical ROC analysis of Type 2 data, as described previously (Critchfield, 1993; Hosseini \& Ferrell, 1982), can be applied without knowledge of the Type 1 distribu- tions, but to make predictions of Type 2 performance based on the principles of TSD, theoretical Type 2 probability functions are required. If it is reasonable to assume that Type 1 and Type 2 decisions are based on the same evidence, our Type 2 equations provide the basis for predicting performance. If the object of an experiment is to discover whether the Type 1 and the Type 2 decisions are based on the same evidence, as in studies of perception without awareness, our equations provide the basis for predicting Type 2 performance under the null hypothesis that Type 1 and Type 2 decisions are based on the same evidence.

\section{REFERENCES}

BANKs, W. P. (1970). Signal detection theory and human memory. Psychological Bulletin, 74, 81-99.

Baranski, J. V., \& Petrusic, W. M. (1998). Probing the locus of confidence judgements: Experiments on the time to determine confidence. Journal of Experimental Psychology: Human Perception \& Performance, 24, 929-945.

BIRDSALL, T. G. (1955). The theory of signal detectability. In H. Quastler (Ed.), Information theory in psychology (pp. 391-402). Glencoe, IL: Free Press.

Busey, T. A., Tunnicliff, J., Loftus, G. R., \& Loftus, E. F. (2000). Accounts of the confidence-accuracy relation in recognition memory. Psychonomic Bulletin \& Review, 7, 26-48.

Clarke, F. R., Birdsall, T. G., \& TANnER, W. P., JR. (1959). Two types of ROC curves and definitions of parameters. Journal of the Acoustical Society of America, 31, 629-630.

CRITChField, T. S. (1993). Signal-detection properties of verbal selfreports. Journal of the Experimental Analysis of Behavior, 60, 495-514.

EGAN, J. P. (1975). Signal detection theory and ROC analysis. New York: Academic Press.

EGAN, J. P., \& CLARKE, F. R. (1956). Source and receiver behavior in the use of a criterion. Journal of the Acoustical Society of America, 28, 1267-1269.

Egan, J. P., Clarke, F. R., \& Carterette, E. C. (1956). On the transmission and confirmation of messages in noise. Journal of the Acoustical Society of America, 28, 536-550.

Ferrell, W. R., \& MCGoey, P. J. (1980). A model of calibration for subjective probabilities. Organizational Behavior \& Human Performance, 26, 32-53.

GALVIN, S. J. (1988). The theory of Type II ROC analysis. Unpublished master's thesis, Victoria University, Wellington, New Zealand.

Galvin, S. J., Beuke, C. J., KeOGH, D., \& Robinson, T. (2000). Can utrocular discrimination be subliminal? Investigative Ophthalmology \& Visual Science, 41(Suppl.), S732.

GigerenZer, G., Hoffrage, U., \& Kleinbölting, H. (1991). Probabilistic mental models: A Brunswikian theory of confidence. Psychological Review, 98, 506-528.

GreEN, D. M., \& SwETS, J. A. (1966). Signal detection theory and psychophysics. New York: Wiley.

Healy, A. F., \& Jones, C. (1973). Criterion shifts in recall. Psychological Bulletin, 79, 335-340.

Higham, P. A. (2002). Strong cues are not necessarily weak: Thomson and Tulving (1970) and the encoding specificity principle revisited. Memory \& Cognition, 30, 67-80.

Hosse INI, J., \& FerReLl, W. R. (1982). Detectability of correctness: A measure of knowing that one knows. Instructional Science, 11, 113-127.

KEREN, G. (1988). On the ability of monitoring non-veridical perceptions and uncertain knowledge: Some calibration studies. Acta Psychologica, 67, 95-119.

KEREN, G. (1991). Calibration and probability judgements: Conceptual and methodological issues. Acta Psychologica, 77, 217-273.

Kolb, F. C., \& BraUn, J. (1995). Blindsight in normal observers. Nature, 377, 336-338.

Kunimoto, C., Miller, J., \& PAshler, H. (2001). Confidence and accuracy of near-threshold discrimination responses. Consciousness \& Cognition, 10, 294-340.

Lichtenstein, S., FischHoff, B., \& Phillips, L. D. (1982). Calibration 
of probabilities: The state of the art to 1980. In D. Kahneman, P. Slovic, \& A. Tversky (Eds.), Judgement under uncertainty: Heuristics and biases (pp. 306-334). Cambridge: Cambridge University Press.

LINK, S. W. (1992). The wave theory of difference and similarity. Hillsdale, NJ: Erlbaum.

LUCE, R. D. (1997). Some unresolved conceptual problems in mathematical psychology. Journal of Mathematical Psychology, 41, 79-87.

Macmillan, N. A., \& Creelman, C. D. (1991). Detection theory: A user's guide. Cambridge: Cambridge University Press.

MCFADDEN,D., \& GREENO,J. G. (1966). Verbal learning: A some-or-none process (Tech. Report). Indiana University Hearing and Communication Laboratory.

Merikle, P. M., Smilek, D., \& Eastwood, J. D. (2001). Perception without awareness: Perspectives from cognitive psychology. Cognition, 79, 115-134.

Mood, A. M., Graybill, F. A., \& Boes, D. C. (1974). Introduction to the theory of statistics (3rd ed., International Student Edition). Singapore: McGraw-Hill.

MURDOCK, B. B., JR. (1966). The criterion problem in short term memory. Journal of Experimental Psychology, 72, 317-324.

MUrdock, B. B., JR. (1974). Human memory: Theory and data. Potomac, MD: Erlbaum.

Norman, D. A., \& Wickelgren, W. (1969). Strength theory of decision rules and latency in retrieval from short-term memory. Journal of Mathematical Psychology, 6, 192-208.

PEIRCE, C. S., \& JASTROW, J. (1884). On small differences in sensation. Memoirs of the National Academy of Sciences, 3, 73-83.

PoDD, J. V. (1975). Type I and Type II ROC analysis of change in human decision axis. Unpublished master's thesis, Victoria University, Wellington, New Zealand.

POLlaCK, I. (1959). On indices of signal and response discriminability. Journal of the Acoustical Society of America, 31, 1031.

POLlACK, I., \& DECKER, L. R. (1958). Confidence ratings, message reception, and the receiver operating characteristic. Journal of the Acoustical Society of America, 30, 286-292.

Reber, A. S., Kassin, S. M., Lewis, S., \& CANTor, G. W. (1980). On the relationship between implicit and explicit modes in the learning of a complex rule structure. Journal of Experimental Psychology: Human Learning \& Memory, 6, 492-502.

SWETS, J. A. (1973). The relative operating characteristic in psychology. Science, 182, 990-1000.

Swets, J. A., TANner, W. P., JR., \& Birdsall, T. G. (1961). Decision processes in perception. Psychological Review, 68, 301-340.

TAYLOR, A., BOVEN, R., \& WHITMORE, J. (1991). Reduction of unique noise in the psychophysics of hearing by group operating characteristic analysis. Psychological Bulletin, 109, 133-146.

VAN ZANDT, T. (2000). ROC curves and confidence judgements in recognition memory. Journal of Experimental Psychology: Learning, Memory, \& Cognition, 26, 582-600.

VICKERS, D. (1979). Decision processes in visual perception. New York: Academic Press.

We ISKRANTZ, L. (1986). Blindsight: A case study and its implications. Oxford: Oxford University Press.

Weiskrantz, L. (1998). Consciousness and commentaries. International Journal of Psychology, 33, 227-233.

\section{NOTES}

1. The term probability function here refers to either a continuous probability density function or a discrete mass function.
2. In this context, a Type 2 rating would be one that followed a binary Type 1 decision about whether the word was old or new. The Type 2 rating would record the observer's confidence that the Type 1 binary response had been correct. That was not the procedure used in Banks's (1970) experiment.

3. For the same reason, we prefer the original theory of signal detectability to the now common signal detection theory (SDT). We also wish to avoid the assumption sometimes attached to the label SDT that the distributions of the evidence are Gaussian.

4. Clarke et al. (1959) used the terms stimulus-conditional and response-conditional to refer to the Type 1 and Type 2 tasks, respectively. We have avoided the use of these terms because (1) both decisions are made on the basis of the stimulus, as we describe here, and (2) the terms stimulus-conditional and response-conditional are sometimes used to distinguish classical from operant conditioning; this distinction is not related to that between Type 1 and Type 2 tasks.

5. Making an incorrect Type 2 decision should not be confused with the Type 2 error of hypothesis testing. Although there is a relationship between TSD and the logic of hypothesis testing (see Swets, 1973), the use of the term Type 2 in both systems does not reflect that relationship.

6 . In this article, we consider decision rules that involve applying a single criterion to some decision axis, and that axis may be $X$, or some transformation of $X$. Therefore, in a situation in which one can produce the same set of responses by applying either a single criterion to the likelihood ratio axis or more than one criterion to the $X$ axis, we describe this as using the likelihood ratio of $X, \operatorname{not} X$, as the decision axis.

7. In the case of a model based on a Type 1 decision axis $V=t_{1}(X)$, the equations applied below to the Type 1 probability functions $f(x \mid S)$ and $f(x \mid N)$ should instead be applied to the probability functions of the transformed variable, $f(v \mid S)$ and $f(v \mid N)$, respectively.

8. Cumulative distribution functions of $X$ give the area under the probability function, $f(x)$, to the left of each value of $X$-that is, $F(k)=\int_{-\infty}^{k}$ $f(x) d x$. For discrete functions, $F(k)=P(X \leq k)$.

9. Most authors would use the decision rule "Say 'yes' if $x$ is greater than or equal to $k$." For discrete probability functions, Equations 1 and 2 would then not hold. Our change to the decision rule is made for convenience in the discrete case and does not affect the generality of our results in either the discrete or the continuous case.

10. Green and Swets (1966) showed that the Type 1 likelihood ratio is the optimal decision axis for Type 1 decisions for several decision goals, regardless of the underlying Type 1 probability functions. The same explanation applies to the likelihood ratio for Type 2 probability functions.

11. Although it makes no difference to the mathematics, we switch to using the variable $x$ rather than $m$ at this point, since we are now thinking of the variable as representing values of evidence, rather than as Type 2 criteria applied to the $X$ axis.

12. In the two-interval task, the observer is presented with evidence sampled from the $S$ distribution in one spatial or temporal observation interval, and evidence sampled from the $N$ distribution in the other interval. The observer's task is to say in which interval (first or second, left or right, etc.) the signal event occurred. This task is also known as twoalternative forced choice, but because an observer is also forced to choose between two alternatives in the yes-no task, we prefer to avoid the ambiguity by using the term two-interval.

13. Other decision variables might be used, such as $W=X_{1} / X_{2}$.

14. See Taylor, Boven, and Whitmore (1991) for a discussion of common and unique noise.

15. Type 2 ROC analysis can also be used on decisions that assess the correctness of multiple-interval tasks (e.g., Kolb \& Braun, 1995), but we have not presented a model for that here. 


\section{APPENDIX A \\ The Type 2 Theory of Clarke, Birdsall, and Tanner (1959)}

Clarke et al. (1959) presented the only prior attempt we have seen to derive a pair of Type 2 probability density functions. They considered the case of the likelihood ratio observer's attempting to detect a signal known exactly in a background of white, Gaussian noise (see Green \& Swets, 1966, for details about this task). In this case, the Type 1 density function of the evidence variable, $X$, is normal for each of the $S$ and $N$ events, and both functions have the same variance. The separation of the means in standard deviation units is $d_{X}^{\prime}$.

Clarke et al. (1959) transformed the Type 1 evidence axis, $X$, using $Y=\log [l(X)] / d_{X}^{\prime}$. The density functions of $Y$ conditional on $S$ and $N$ can be shown to be normal, with unit variance and with means at $\pm d_{X}^{\prime} / 2$. We refer to these density functions as $g(y \mid S)$ and $g(y \mid N)$. Note that $d_{Y}^{\prime}$ for the probability density functions of $Y$ conditional on $S$ and $N$ is the same as $d_{X}^{\prime}$. This is expected, since $Y$ is a linear increasing transformation of $X$. The reason for the transformation was not, therefore, to obtain an optimal decision axis, because $X$ is already that; the advantage it confers is to simplify the mathematics of deriving their Type 2 model. In particular, the functions $g(y \mid S)$ and $g(y \mid N)$ intersect at zero, giving $l(Y)>1$ for positive values of $Y$ only.

Clarke et al. (1959) made the important point that the Type 2 functions would depend on the value of $Y$ used as the criterion in the Type 1 decision. They chose the optimal criterion for the Type 1 observer-namely, the $Y$ value correspondingto an $l(Y)$ value of 1 [because the Type 1 priors, $P(S)$ and $P(N)$, were set to one half]. If $X$ is used as the decision axis, this criterion is some value of $X$ (call it $x_{0}$ ); if $Y$ is the decision axis, the criterion is $y=0$.

Clarke et al. (1959) wrote that they were going to use a likelihood ratio as the decision axis for the Type 2 decision, $l_{r}(x)=P_{c}(x) / P_{I}(x)$, where $r$ stands for response conditional (meaning Type 2 ). Although Clarke et al. used $X$ as the variable in that expression, we have taken this likelihood ratio to correspond to our $l_{0}(Y)$, the ratio of the Type 2 probability functions $g_{0}(y \mid C)$ and $g_{0}(y \mid I)$. These are obtained by applying the Type 1 criterion $y=0$ to $g_{0}(y \mid S)$ and $g_{0}(y \mid N)$, and this is how they described what they were doing. Also, the rest of their derivation is expressed as functions of $Y$, not of $X$. In fact, the density functions of $l_{\mathrm{x}_{0}}(X)$ and $l_{0}(Y)$ conditional on $C$ and $I$ will produce the same ROC curve, because $X$ and $Y$ increase strictly monotonically together.

At this point Clarke et al. (1959) wrote, "In this example, the decision rule for making identification responses results in $l_{r}(x)=l(x)$ for $y>0$ and $l_{r}(x)=1 / l(x)$ for $y<0$." We now know that, even for equal Type 1 prior probabilities, these equations are out by a scale factor of $P_{k}(I) / P_{k}(C)$; see Equations B8a and B8b in the proof of Corollary 2). However, $l_{r}(x)$ is proportional to $l(x)$ for $y>0$ and to $1 / l(X)$ for $y<0$. From this relationship between the Type 1 and the Type 2 likelihood ratios, Clarke et al. concluded that the probability density functions conditional on $C$ and $I$ for the likelihood ratio observer would be the probability density functions of $W$ conditional on $C$ and $I$, where $W=|Y|\left\{\right.$ recall that $\left.Y=\log [l(X)] / d_{X}^{\prime}\right\}$. They obtained the probability density functions of $|Y|$ by scaling up parts of $g(y \mid S)$ and $g(y \mid N)$ for positive values of $Y$ so that the area under each was 1 . This gave the same result that we get by applying our equations for the Type 2 decision axis of $|X-k|$ for $k=0$ (see Corollary 4 ) to the probability functions of $Y$-namely (in our notation),

$$
f_{0}(w \mid C)=\frac{1}{P_{0}(C) \sqrt{2 \pi}} \exp \left[-\frac{1}{2}\left(w-\frac{d^{\prime}}{2}\right)^{2}\right], w>0,
$$

and

$$
f_{0}(w \mid I)=\frac{1}{P_{0}(I) \sqrt{2 \pi}} \exp \left[-\frac{1}{2}\left(w-\frac{d^{\prime}}{2}\right)^{2}\right], w>0,
$$

Although Clarke et al. (1959) correctly derived the Type 2 probability density functions for the decision axis $W=|Y|$, that is not actually what they set out to do, and we will show below that those functions are different from the Type 2 probability density functions for the intended decision axis, $l_{0}(Y)$. It is true, however, that although $|Y|$ and $l_{0}(Y)$ do not have the same Type 2 probability functions, they do give the same Type 2 ROC curves in this case, because $|Y|$ varies monotonically with $l_{0}(Y)$ for this particular choice of Type 1 probability functions and Type 1 criterion.

We derive the Type 2 functions for $l_{0}(Y)$ by applying the equations in Table 4 to the probability functions of the Type 1 decision variable, $g(y \mid S)$ and $g(y \mid N)$, to obtain the Type 2 functions based on decision axis $Y, g_{0}(y \mid C)$ and $g_{0}(y \mid I)$. We use these probability functions to calculate how the transformation of $Y, l_{0}(Y)$, is distributed conditional on $C$ and $I$.

The Type 1 density functions of $Y$ are given as

$$
g(y \mid S)=\frac{1}{\sqrt{2 \pi}} \exp \left[-\frac{1}{2}\left(y-\frac{d^{\prime}}{2}\right)^{2}\right]
$$

and

$$
g(y \mid N)=\frac{1}{\sqrt{2 \pi}} \exp \left[-\frac{1}{2}\left(y+\frac{d^{\prime}}{2}\right)^{2}\right] .
$$




\section{APPENDIX A (Continued)}

From the equations in Table 4, the Type 2 probability density functions of $Y$, given $y=0$ as the Type 1 criterion and $P(S)=P(N)=.5$, are

$$
g_{0}(y \mid C)=\left\{\begin{array}{l}
\frac{1}{2 P_{0}(C)} \cdot \frac{1}{\sqrt{2 \pi}} \exp \left[-\frac{1}{2}\left(y+\frac{d^{\prime}}{2}\right)^{2}\right], \text { for } y \leq 0 \\
\frac{1}{2 P_{0}(C)} \cdot \frac{1}{\sqrt{2 \pi}} \exp \left[-\frac{1}{2}\left(y+\frac{d^{\prime}}{2}\right)^{2}\right], \text { for } y>0
\end{array}\right.
$$

and

$$
g_{o}(y \mid I)=\left\{\begin{array}{l}
\frac{1}{2 P_{0}(I)} \cdot \frac{1}{\sqrt{2 \pi}} \exp \left[-\frac{1}{2}\left(y-\frac{d^{\prime}}{2}\right)^{2}\right], \text { for } y \leq 0 \\
\frac{1}{2 P_{0}(I)} \cdot \frac{1}{\sqrt{2 \pi}} \exp \left[-\frac{1}{2}\left(y+\frac{d^{\prime}}{2}\right)^{2}\right], \text { for } y>0
\end{array}\right.
$$

for equal priors. The Type 2 likelihood ratio for these functions is

$$
\begin{aligned}
l_{0}(y) & =\frac{g_{0}(y \mid C)}{g_{0}(y \mid I)} \\
& =\left\{\begin{array}{l}
\frac{g_{0}(y \mid N)}{g_{0}(y \mid S)} \cdot \frac{P_{0}(I)}{g_{0}(C)}, \text { for } y \leq 0 \\
\frac{g_{0}(y \mid S)}{g_{0}(y \mid N)} \cdot \frac{P_{0}(I)}{P_{0}(C)}, \text { for } y>0
\end{array}\right. \\
& =\left\{\begin{array}{l}
\exp \left\{-\frac{1}{2}\left[\left(y+\frac{d^{\prime}}{2}\right)^{2}-\left(y-\frac{d^{\prime}}{2}\right)^{2}\right]\right\} \frac{P_{0}(I)}{P_{0}(C)}, \text { for } y \leq 0 \\
\exp \left\{-\frac{1}{2}\left[\left(y-\frac{d^{\prime}}{2}\right)^{2}-\left(y+\frac{d^{\prime}}{2}\right)^{2}\right]\right\} \frac{P_{0}(I)}{P_{0}(C)}, \text { for } y>0
\end{array}\right. \\
& =\left\{\begin{array}{l}
\exp \left(-y d^{\prime}\right) \frac{P_{0}(I)}{P_{0}(C)}, \text { for } y \leq 0 \\
\exp \left(y d^{\prime}\right) \frac{P_{0}(I)}{P_{0}(C)}, \text { for } y>0 .
\end{array}\right.
\end{aligned}
$$

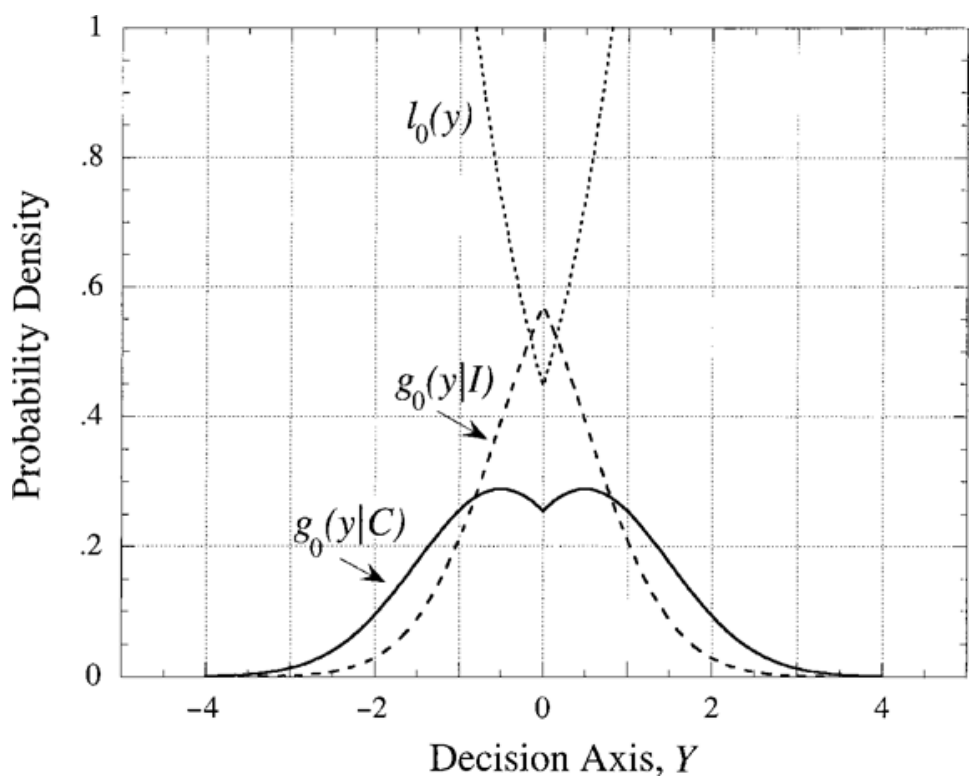

Figure A1. Type 2 probability density functions of $Y$ for $k=0$ and $d^{\prime}=1$ and their likelihood ratio. 


\section{APPENDIX A (Continued)}

The distributions $g_{0}(y \mid C)$ and $g_{0}(y \mid I)$ and their likelihood ratio are shown in Figure A1.

We now derive the probability density functions of the likelihood ratio, $l_{0}(Y)$, conditional on $C$ and $I$ by a transformation of variable. In general, if $Y$ has probability density function $g(y)$, let $\boldsymbol{\aleph}=$ $\{y: g(y)>0\}$; if $z=t(y)$ defines a one-to-one transformation of $\boldsymbol{\aleph}$ onto $\mathfrak{i}$ and

$$
\frac{d\left[t^{-1}(z)\right]}{d z}
$$

is continuous and nonzero for $z \in \mathfrak{R}, Z$ has probability density function

$$
h(z)=\left|\frac{d}{d z} t^{-1}(z)\right| \cdot g\left[t^{-1}(z)\right], \text { for } z \in \Re,
$$

and $h(z)=0$ elsewhere (Mood, Graybill, \& Boes, 1974, p. 200). (We are using the variable $Z$ here to distinguish this transformation from the transformation $W=|Y|$; it has nothing to do with $Z$ scores.) Since the transformation function, $\mathrm{z}=l_{0}(y)$ is many to one (see Figure A1), $\aleph$ must be broken into disjoint sets, $\boldsymbol{\aleph}_{1} \ldots \boldsymbol{\aleph}_{n}$, in such a way that $z=t(y)$ defines a one-to-one transformation of each $\aleph_{i}$ onto $\Re$. Then the density of $Y$ given by

$$
h(z)=\sum\left|\frac{d}{d z} t_{i}^{-1}(z)\right| \cdot g\left[t_{i}^{-1}(z)\right], \text { for } z \in \Re,
$$

where the summation is over those values of $i$ for which $t(y)=z$ for some value of $y$ in $\aleph_{i}$ (Mood et al., 1974, p. 201). For the transformation function $z=l_{0}(y)$, the positive and negative regions of $Y$ each support one-to-one functions, so the probability functions of $l_{0}(Y)$ conditional on $C$ and $I$ are the sum of contributions from these two regions.

The transformation function is different for positive and negative regions of $Y$, so the inverse of the transformation function is calculated separately for each, as follows:

$$
\begin{aligned}
& \text { Let } z=t_{1}(y)=\exp \left(y d^{\prime}\right) \frac{P_{0}(I)}{P_{0}(C)} \text {, for } y>0 \text {. Then, } \\
& \qquad y=t_{i}^{-1}(z)=\frac{1}{d^{\prime}} \ln \left(\frac{P_{0}(C)}{P_{0}(I)} \cdot z\right), \text { for } t_{i}^{-1}(z)>0
\end{aligned}
$$

- that is,

$$
\text { for } \frac{1}{d^{\prime}} \ln \left(\frac{P_{0}(C)}{P_{0}(I)} \cdot z\right)>0
$$

giving

$$
\frac{P_{0}(C)}{P_{0}(I)} \cdot z>1
$$

or

$$
z>\frac{P_{0}(I)}{P_{0}(C)} .
$$

Also,

$$
\frac{d}{d z}\left[t_{i}^{-1}(z)\right]=\frac{1}{d^{\prime} z}, \quad \text { for } z>\frac{P_{0}(I)}{P_{0}(C)} .
$$

The contribution to $h_{0}(z \mid C)$, the probability density function of $l_{0}(Y)$ for the region $y>0$ is, therefore,

$$
\begin{aligned}
& g_{0}\left[t_{i}^{-1}(z) \mid C\right]\left|\frac{d}{d z} t_{i}^{-1}(z)\right|, \quad \text { for } t_{i}^{-1}(z)>0 \\
& =\frac{1}{2 P_{0}(C)} g\left[t_{i}^{-1}(z) \mid S\right] \frac{1}{d^{\prime} z} \text {, for } z>\frac{P_{0}(I)}{P_{0}(C)}, \text { from Equations A3b and A6 } \\
& =\frac{1}{2 P_{0}(C)} \cdot \frac{1}{\sqrt{2 \pi}} \cdot \frac{1}{d^{\prime} z} \exp \left\{-\frac{1}{2}\left[\frac{1}{d^{\prime}} \ln \left(\frac{P_{0}(C)}{P_{0}(I)} \cdot z\right)-\frac{d^{\prime}}{2}\right]^{2}\right\}, \text { for } z>\frac{P_{0}(I)}{P_{0}(C)}, \text { from Equation A5. }
\end{aligned}
$$

Now let

$$
z=t_{2}(y)=\exp \left(-y d^{\prime}\right) \frac{P_{0}(I)}{P_{0}(C)}, \text { for } y \leq 0
$$


Then,

$$
y=t_{2}^{-1}(z)=-\frac{1}{d^{\prime}} \ln \left(\frac{P_{0}(C)}{P_{0}(I)} \cdot z\right), \text { for } t_{2}^{-1}(z) \leq 0
$$

that is,

giving

$$
\frac{1}{d^{\prime}} \ln \left(\frac{P_{0}(C)}{P_{0}(I)} \cdot z\right) \geq 0
$$

as before, and

$$
z>\frac{P_{0}(I)}{P_{0}(C)}
$$

$$
\frac{d}{d z}\left[t_{2}^{-1}(z)\right]=\frac{-1}{d^{\prime} z}, \quad \text { for } z \geq \frac{P_{0}(I)}{P_{0}(C)} .
$$

The contribution to $h_{0}(z \mid C)$, the probability density function of $l_{0}(Y)$, for the region $y \leq 0$ is, therefore,

$$
\begin{aligned}
& g_{0}\left(t_{2}^{-1}(z) \mid C\right)\left|\frac{d}{d z}\left[t_{2}^{-1}(z)\right]\right|, \text { for } t_{2}^{-1}(z) \leq 0 \\
& =\frac{1}{2 P_{0}(C)} g\left(t_{2}^{-1}(z) \mid N\right) \frac{1}{d^{\prime} z}, \text { for } z \geq \frac{P_{0}(I)}{P_{0}(C)}, \text { from Equations A3a and A8 } \\
& =\frac{1}{2 P_{0}(C)} \cdot \frac{1}{\sqrt{2 \pi}} \cdot \frac{1}{d^{\prime} z} \cdot \exp \left\{-\frac{1}{2}\left[-\frac{1}{d^{\prime}} \ln \left(\frac{P_{0}(C)}{P_{0}(I)} \cdot z\right)+\frac{d^{\prime}}{2}\right]^{2}\right\}, \text { for } z \geq \frac{P_{0}(I)}{P_{0}(C)}, \text { from Equation A7, }
\end{aligned}
$$

which is the same as the contribution from $t_{1}(Y)$.

The probability density function of $Z$ [i.e., of $l_{0}(Y)$ ] conditional on $C$ is, therefore,

$$
h_{0}(z \mid C)=\frac{1}{P_{0}(C)} \cdot \frac{1}{\sqrt{2 \pi}} \cdot \frac{1}{d^{\prime} z} \cdot \exp \left\{-\frac{1}{2}\left[\frac{1}{d^{\prime}} \ln \left(\frac{P_{0}(C)}{P_{0}(I)} \cdot z\right)-\frac{d^{\prime}}{2}\right]^{2}\right\}, \text { for } z>\frac{P_{0}(I)}{P_{0}(C)} .
$$

The contribution to $h_{0}(z \mid I)$ for the region $y>0$ is

$$
\begin{gathered}
g_{0}\left(t_{i}^{-1}(z) \mid I\right)\left|\frac{d}{d z}\left[t_{i}^{-1}(z)\right]\right|, \quad \text { for } t_{1}^{-1}(z)>0 \\
=\frac{1}{2 P_{0}(I)} g\left(t_{i}^{-1}(z) \mid N\right) \frac{1}{d^{\prime} z}, \text { for } z>\frac{P_{0}(I)}{P_{0}(C)}, \text { from Equations A4b and A6 } \\
=\frac{1}{2 P_{0}(I)} \cdot \frac{1}{\sqrt{2 \pi}} \cdot \frac{1}{d^{\prime} z} \exp \left\{-\frac{1}{2}\left[\frac{1}{d^{\prime}} \ln \left(\frac{P_{0}(C)}{P_{0}(I)} \cdot z\right)+\frac{d^{\prime}}{2}\right]^{2}\right\}, \text { for } z>\frac{P_{0}(I)}{P_{0}(C)}, \text { from Equation A5, }
\end{gathered}
$$

and the contribution from the region $y \leq 0$ is

$$
\begin{aligned}
& g_{0}\left(t_{2}^{-1}(z) \mid I\right)\left|\frac{d}{d z}\left[t_{2}^{-1}(z)\right]\right|, \quad \text { for } t_{2}^{-1}(z) \leq 0 \\
& =\frac{1}{2 P_{0}(C)} g\left(t_{2}^{-1}(z) \mid S\right) \frac{1}{d^{\prime} z}, \text { for } z \geq \frac{P_{0}(I)}{P_{0}(C)}, \text { from Equations A4a and A8 } \\
& =\frac{1}{2 P_{0}(C)} \cdot \frac{1}{\sqrt{2 \pi}} \cdot \frac{1}{d^{\prime} z} \exp \left\{-\frac{1}{2}\left[-\frac{1}{d^{\prime}} \ln \left(\frac{P_{0}(C)}{P_{0}(I)} \cdot z\right)-\frac{d^{\prime}}{2}\right]^{2}\right\}, \text { for } z \geq \frac{P_{0}(I)}{P_{0}(C)}, \text { from Equation A7. }
\end{aligned}
$$

The probability density function of $Z$ [i.e., of $l_{0}(Y)$ ] conditional on $I$ is, therefore,

$$
h_{0}(z \mid I)=\frac{1}{P_{0}(I)} \cdot \frac{1}{\sqrt{2 \pi}} \cdot \frac{1}{d^{\prime} z} \cdot \exp \left\{-\frac{1}{2}\left[\frac{1}{d^{\prime}} \ln \left(\frac{P_{0}(C)}{P_{0}(I)} \cdot z\right)-\frac{d^{\prime}}{2}\right]^{2}\right\}, \text { for } z>\frac{P_{0}(I)}{P_{0}(C)}
$$


APPENDIX A (Continued)

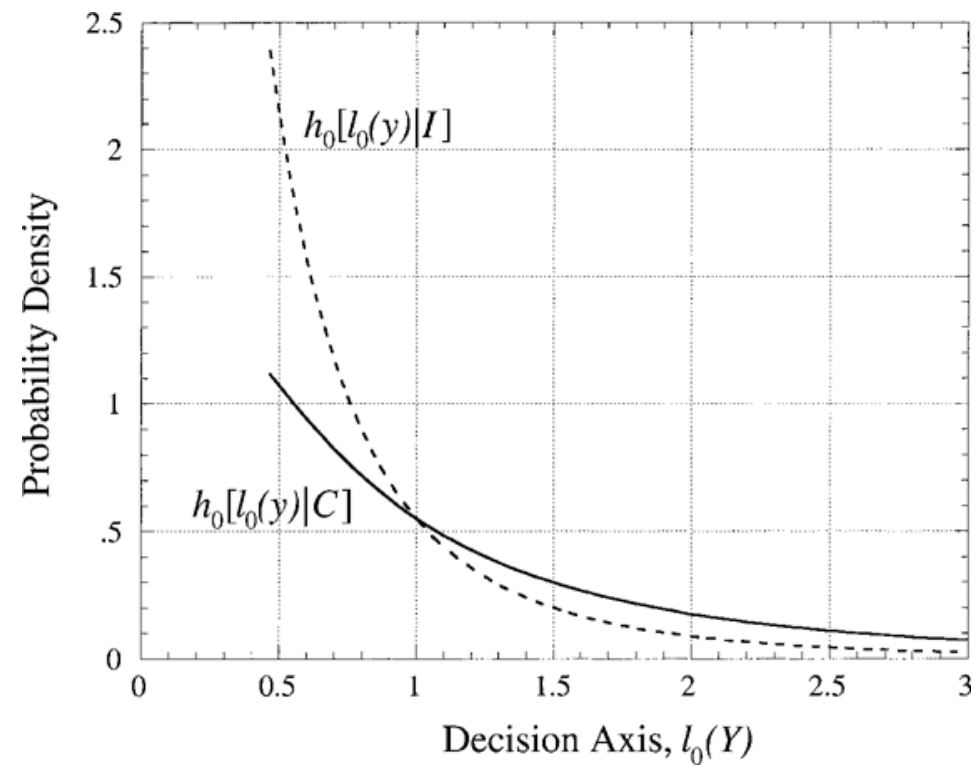

Figure A2. Type 2 probability density functions of $l_{0}(Y)$, where the Type 1 functions of $Y$ are normal with a variance of 1 and with means at +0.5 and -0.5 , respectively.

The integrals under each of the two Type 2 probability functions of $Z$ can be shown to be equal to one, and the likelihood ratio of the two functions is $Z$, as required of probability density function of likelihood ratios in general. The distributions $h_{0}(y \mid C)$ and $h_{0}(y \mid I)$ are shown in Figure A2.

The distributions $h_{0}(z \mid C)$ and $h_{0}(z \mid I)$ are the model for the Type 2 task described by Clarke et al. (1959) and would produce the same ROC curve as Functions A1 and A2 derived by them. However, $h_{0}(z \mid C)$ and $h_{0}(z \mid I)$ differ from those in Clarke et al. in two important ways. First, they both have a range that starts at a point above zero. From Figure A1, it can be seen that the minimum value of $l_{0}(y)$, which is derived by dividing $g_{0}(y \mid C)$ by $g_{0}(y \mid I)$, occurs at $y=0$; note that the vertical axis in Figure A1 is the same as the horizontal axis in Figure A2. The inequality at the end of the sentence containing Equation A5 shows that this minimum value of $l_{0}(y)$ is $P_{0}(I) / P_{0}(C)$. Second, neither $h_{0}(z \mid C)$ nor $h_{0}(z \mid I)$ is a scaled portion of a Gaussian distribution, as both of the functions in Clarke et al. were. 


\section{APPENDIX B \\ Proofs of Corollaries to the General Equations}

COROLlary 1: Type 2 ROC Curves for Extreme Type 1 Criteria

The 1:X; 2:X ROC curve obtained using the lowest possible Type 1 criterion (i.e., always saying "yes" in the Type 1 task) is the Type 1 ROC curve; the 1:X; 2:X ROC curve obtained using the highest possible criterion in the Type 1 task (i.e., always say "no") is the reflection of the Type 1 ROC curve in the chance line. The $1: X ; 2: l_{k}(X)$ ROC curve obtained using the highest possible criterion in the Type 1 task is the reflection in the negative diagonal of the $1: X ; 2: l_{k}(X)$ ROC curve for the lowest possible criterion.

As the Type 1 criterion yielding the Type 2 ROC curve becomes smaller, the Type 2 curve approaches the Type 1 curve. This can be shown to be true for any pair of Type 1 probability functions. If $k=-\infty$, is taken as the criterion in the Type 1 task, the Type 2 probability functions are completely defined by Equations $10 \mathrm{~b}$ and $11 \mathrm{~b}$, since $x$ is always greater than $k$. For $k=-\infty$, the observer always says "yes" in the Type 1 task, so the probability of being correct is the prior probability of a signal event-that is, $P_{-\infty}(C)=P(S)$ and $P_{-\infty}(I)=P(N)$. Equations $10 \mathrm{~b}$ and $11 \mathrm{~b}$ become

$$
\begin{aligned}
& f_{k}(x \mid C)=f(x \mid S), \text { for } k=-\infty \\
& f_{k}(x \mid I)=f(x \mid N), \text { for } k=-\infty
\end{aligned}
$$

and the Type 2 curve coincides with the Type 1 curve.

If $k=\infty$, the observer always says "no," so $P_{\infty}(C)=P(N)$ and $P_{\infty}(I)=P(S)$. Equations 10a and $11 \mathrm{a}$ are sufficient to give the Type 2 probability functions, which are

$$
\begin{aligned}
& f_{k}(x \mid C)=f(x \mid N), \text { for } k=\infty \\
& f_{k}(x \mid I)=f(x \mid S), \text { for } k=\infty .
\end{aligned}
$$

The Type 2 curve for $k=\infty$ is, therefore, given by $H R_{2}=F A R_{1}$ and $F A R_{2}=H R_{1}$. This exchange of coordinates amounts to a reflection of the Type 1 curve in the line $H R=F A R$, the chance line.

The $1: X ; 2: l_{k}(X)$ ROC curves for the highest and the lowest Type 1 criteria are also reflections of each other, this time in the negative diagonal. The Type 2 likelihood ratios in this case are

$$
l_{-\infty}(x)=l(x), \quad \text { from Equations B1 and B2 }
$$

and

$$
l_{\infty}(x)=[l(x)]^{-1}, \text { from Equations B3 and B4. }
$$

Equations B5 and B6 give

$$
l_{\infty}(x)=\left[l_{-\infty}(x)\right]^{-1},
$$

which gives

$$
l_{-\infty}\left(x_{i}\right)>l_{-\infty}\left(x_{j}\right) \Leftrightarrow l_{\infty}\left(x_{i}\right)<l_{-\infty}(x) .
$$

The Type 2 probability functions for the 1:X; 2: $l_{k}(X)$ case, $g_{k}\left[l_{k}(x) \mid C\right]$ and $g_{k}\left[l_{k}(x) \mid I\right]$, are transformations of the Type 2 probability functions, $f_{k}(x \mid C)$ and $f_{k}(x \mid I)$. From Equations B1 and B4, it can be seen that the functions $g_{-\infty}\left[l_{-\infty}(x) \mid C\right]$ and $g_{\infty}\left[l_{\infty}(x) \mid I\right]$ are both transformations of the function $f(x \mid S)$; Equations B2 and B3 show that $g_{-\infty}\left[l_{-\infty}(x) \mid I\right]$ and $g_{\infty}\left[l_{\infty}(X) \mid C\right]$ are both transformations of $f(x \mid N)$.

For the discrete case, probabilities originally associated with $x$ values become associated with $l_{-\infty}(x)$ values in the $g_{-\infty}\left[l_{-\infty}(x) \mid C\right]$ and $g_{-\infty}\left[l_{-\infty}(x) \mid I\right]$ functions and with $l_{\infty}(x)$ values in the $g_{\infty}$ $\left[l_{\infty}(X) \mid C\right]$ and $g_{\infty}\left[l_{\infty}(x) \mid I\right]$ functions. In the $g_{\infty}\left[l_{\infty}(X) \mid C\right]$ and $g_{\infty}\left[l_{\infty}(x) \mid I\right]$ functions, the order of the probabilities is the reverse of their order in the $g_{-\infty}\left[l_{-\infty}(x) \mid C\right]$ and $g_{-\infty}\left[l_{-\infty}(x) \mid I\right]$ functions, because of the relationship shown in Equation B7. However, for each value $l_{\infty}\left(x_{i}\right)$, there is still a value $l_{-\infty}\left(x_{i}\right)$ equal to $\left[l_{\infty}\left(x_{i}\right)\right]^{-1}$ associated with the same pair of probabilities $P\left(X=x_{i} \mid S\right)$ and $P(X$ $\left.=x_{i} \mid N\right)$. Thus, the functions $g_{-\infty}\left[l_{-\infty}(x) \mid C\right]$ and $g_{\infty}\left[l_{\infty}(x) \mid I\right]$ consist of the same probabilities [those from $f(x \mid S)]$ occurring in opposite orders; $g_{-\infty}\left[l_{-\infty}(x) \mid I\right]$ and $g_{\infty}\left[l_{\infty}(X) \mid C\right]$ are made up of the probabilitiesfrom $f(x \mid N)$, appearing in opposite orders. Therefore, the HR for the Type 2 curve based on $l_{-\infty}(X)$ for some criterion value, $t$, of $l_{-\infty}(X)$ is

$$
\begin{aligned}
H R_{2,-\infty}(t) & =\sum_{\text {values of } l_{-\infty}(x)>t} P_{-\infty}\left[l_{\infty}(X)=l_{-\infty}(x) \mid C\right] \\
& =\sum_{\text {values of } l_{\infty(x)<1 / t}} P_{\infty}\left[l_{\infty}(X)=l_{\infty}(x) \mid I\right] \\
& =1-F A R_{2,-\infty}(\text { first criterionless than } 1 / t) .
\end{aligned}
$$


APPENDIX B (Continued)

Similarly,

$$
\begin{aligned}
F A R_{2,-\infty}(t) & =\sum_{\text {values of } l_{-\infty}(x)>t} P_{-\infty}\left[l_{-\infty}(X)=l_{-\infty}(x) \mid I\right] \\
& =\sum_{\text {values of } l_{\infty}(x)<1 / t} P_{\infty}\left[l_{\infty}(X)=l_{\infty}(x) \mid C\right] \\
& =1-H R_{2,-\infty}(\text { first criterionless than } 1 / t) .
\end{aligned}
$$

For each criterion value on the $l_{-\infty}(X)$ axis, $t$, there is, therefore, a criterion value on the $l_{\infty}(X)$ axis that yields an ROC point on the $\mathrm{ROC}_{2, \infty}$ curve that is the reflection in the negative diagonal of the point yielded by $t$ on the $\mathrm{ROC}_{2, \infty}$ curve.

\section{COROLlaRY 2: Likelihood Ratio for Type 2 Probability Functions}

If $l(X)$ is monotonic with $X$ for $f(x \mid S)$ and $f(x \mid N), l_{k}(X)$ is proportional to $1 / l(x)$ for $x \leq k$ and to $l(x)$ for $x>k$. Hence, $l_{k}(x)$ will be monotonic with $X$ only if $k$ is the lowest possible criterion.

By the equations in Table 4, the likelihood ratios for the Type 2 probability functions (both discrete and continuous) obtained using $k$ as the criterion in the Type 1 decision are given by

$$
\begin{aligned}
l_{k}(x) & =\frac{f_{k}(x \mid C)}{f_{k}(x \mid I)} \\
& = \begin{cases}\frac{f(x \mid N)}{f(x \mid S)} \cdot \frac{P(N)}{P(S)} \cdot \frac{P_{k}(I)}{P_{k}(C)}, \text { for } x \leq k \\
\frac{f(x \mid S)}{f(x \mid N)}, \frac{P(S)}{P(N)} \cdot \frac{P_{k}(I)}{P_{k}(C)}, \text { for } x>k\end{cases} \\
& = \begin{cases}\frac{1}{l(x)} \cdot \frac{P(N)}{P(S)} \cdot \frac{P_{k}(I)}{P_{k}(C)}, \text { for } x \leq k \\
l(x) \cdot \frac{P(S)}{P(N)} \cdot \frac{P_{k}(I)}{P_{k}(C)}, \text { for } x>k .\end{cases}
\end{aligned}
$$

Equation B8b ensures that if $l(X)$ increases monotonically with $X, l_{k}(X)$ will also increase monotonically with $X$ for $x>k$, since $l_{k}(x)$ is equal to $l(x)$ scaled by a constant for a given $k$. Also, by Equation B8a, $l_{k}(x)$ will decrease monotonically with increasing $X$ for $x \leq k$, since $l(x) \geq 0$ and the function $z=1 / y$ decreases for $y>0$. Clearly, if $l(X)$ increases monotonically with $X, l_{k}(X)$ will not increase monotonically with $X$ for all $x$ unless $k=-\infty$.

\section{COROLlary 3: Type 1 Curve as an Upper Bound for Type 2 ROC Curves}

If $l(X)$ is strictly monotonicallyincreasing with $X$, and $P(S)$ and $P(N)$ are equal, the Type 1 ROC curve puts an upper bound on all the Type 2 ROC curves for which $X$ is the decision axis.

Let $k$ be the criterion on the $X$ axis that gives a pair of Type 2 probability functions. Let $x_{1}$ be a criterion for the Type 1 decision and $x_{2}$ be the criterion for the Type 2 decision giving the same FAR as $x_{1}$ gives in the Type 1 task. Let $H R_{1}\left(x_{1}\right)$ be the $H R$ obtained using $x_{1}$ as the criterion in the Type 1 decision and $H R_{2}\left(x_{2}\right)$ be the $H R$ for the Type 2 decision, using $x_{2}$ as the criterion. All the Type 2 curves that can be derived from $f(x \mid S)$ and $f(x \mid N)$ will lie on or below the Type 1 curve if it is true that

$$
H R_{1}\left(x_{1}\right) \geq H R_{2}\left(x_{2}\right), \quad \forall x_{1}, x_{2}, k
$$

Figure B1 illustrates this condition.

For an ROC curve based on likelihood ratio, $H R$ is a strictly monotonically increasing function of $F A R$, and the slope of the curve is monotonically decreasing (Green \& Swets, 1966). This means that the ROC points all lie on or above the chance line $(H R=F A R)$. The following proof of Equation B9 assumes that $l(X)$ increases strictly monotonically with $X$, so the Type 1 curve based on $X$ will be such that

$$
H R_{1}\left(x_{1}\right) \geq F A R_{1}\left(x_{1}\right), \quad \forall x_{1} .
$$

Equal Type1 prior probabilities are also assumed. 
APPENDIX B (Continued)

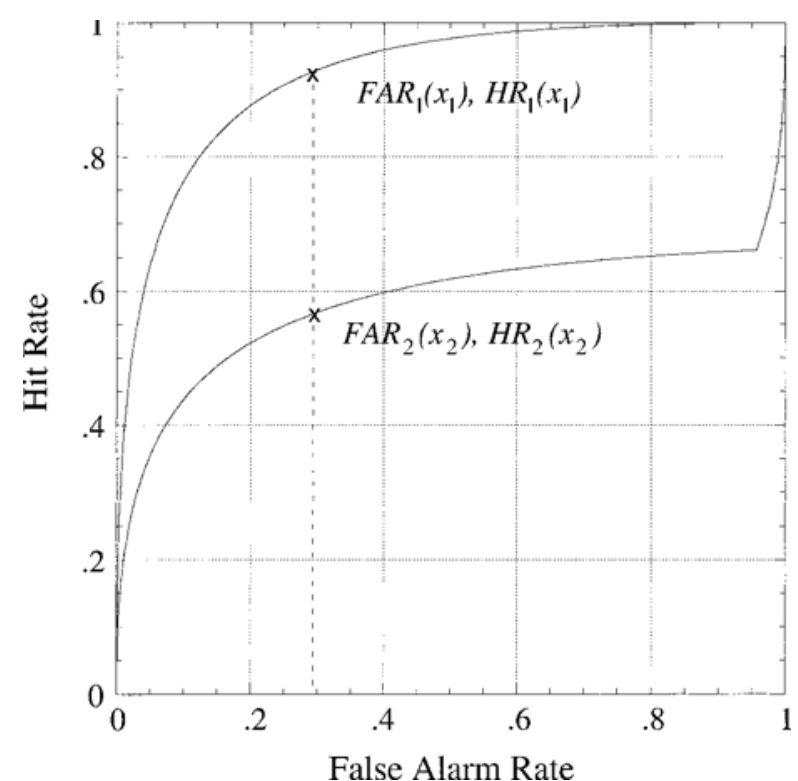

Figure B1. Type 1 receiver operating characteristic (ROC) curve and Type 2 ROC curve showing a pair of points with the same false alarm rate.

Since the equations for $H R_{2}$ and $F A R_{2}$ are different for $x_{2} \leq k$ and $x_{2}>k$, the proof is in two parts. For $x_{2}>k$, equating the $F A R \mathrm{~s}$ gives

$$
1-F\left(x_{1} \mid N\right)=\frac{1-F\left(x_{2} \mid N\right)}{2 P_{k}(I)}, \text { from Equation } 7 \mathrm{~b},
$$

as $P(N)=.5$, so

$$
\left[1-F\left(x_{1} \mid N\right)\right] \cdot 2 P_{k}(I)=1-F\left(x_{2} \mid N\right) .
$$

For the ROC curve with monotonically decreasing slope,

$$
H R(k) \geq F A R(k)
$$

so

$$
H R(k)-F A R(k)+1 \geq 1,
$$

and

$$
.5[H R(k)+1-F A R(k)] \geq .5,
$$

giving

$$
P_{k}(C) \geq .5 \text { by Equations } 4,1, \text { and } 2 .
$$

For equal priors, therefore,

$$
.5 \leq P_{k}(C) \leq 1,
$$

giving

$$
1 \leq 2 P_{k}(C) \leq 2 .
$$

This also gives

$$
2-1 \geq 2-2 P_{k}(C) \geq 2-2,
$$

or

$$
1 \geq 2 P_{k}(I) \geq 0 .
$$

From Equations B10 and B12,

$$
1-F\left(x_{1} \mid N\right) \geq 1-F\left(x_{2} \mid N\right),
$$


which implies

$$
\begin{aligned}
& x_{1} \leq x_{2} \\
& \Rightarrow 1-F\left(x_{1} \mid S\right) \geq 1-F\left(x_{2} \mid S\right) \\
& \Rightarrow 1-F\left(x_{1} \mid S\right) \geq \frac{1-F\left(x_{2} \mid S\right)}{2 P_{k}(C)}, \text { by Equation B11 }
\end{aligned}
$$

and

as required.

$$
H R_{1}\left(x_{1}\right) \geq H R_{2}\left(x_{2}\right),
$$

It can be shown that the point on the $1: X ; 2: X$ ROC curve obtained by using $k$ as the Type 1 criterion lies at the upper end of the part of the curve obtained from Type 2 criterion values $x>k$. The upper segment of the curve (that for criterion values $x \leq k$ ) joins the point $\left[F A R_{2, k}(k), H R_{2, k}(k)\right]$ to the top right corner of the ROC space. For this part of the curve, the slope, given by $l_{k}(x)$, decreases with increasing $x$ (see Equation B8a) - that is, the slope of the curve increases with increasing FAR. Because the slope is always positive (because the likelihood ratio is always positive) and monotonically increasing, this part of the Type 2 curve is always concave upward. It will intersect the Type 1 curve, which is concave downward, at only one point-namely, $(1,1)$. Although two curves of opposite concavity may intersect twice, the bottom end of the upper segment of the Type 2 curve lies on or below the Type 1 curve. This means that no part of this segment lies above the Type 1 curve, as is required.

A similar argument will also show that all the Type 2 curves of a $1: X ; 2: X$ family [with $X$ strictly monotonic with $l(X)$ and equal Type 1 priors] lie above the Type 2 curve yielded by the greatest possible Type 1 criterion. The whole family is, therefore, bounded by the Type 1 curve and its reflection in the chance line (by Corollary 1 ).

\section{Corollary 4: Distance From Type 1 Criterion as Type 2 Decision Axis}

The Type 2 probability functions of the decision variable $W=t(X)$ can be obtained from the Type 2 functions of $X, f_{k}(x \mid C)$, and $f_{k}(x \mid I)$, using

$$
h(w)=\sum\left|\frac{d}{d w} t_{i}^{-1}(w)\right| \cdot f\left[t_{i}^{-1}(w)\right]
$$

where the summation is over separate regions (indexed by $i$ ) for which $w=t(x)$ is a one-to-one transformation, as is explained in Appendix A. If the Type 2 decision is based on the distance between the evidence and the criterion, $k$, the appropriate transformation function is $W=|X-k|$, or

$$
\begin{aligned}
w & =t(x) \\
& =|x-k| \\
& =\left\{\begin{array}{l}
k-x, x \leq k \\
x-k, x>k .
\end{array}\right.
\end{aligned}
$$

The inverse of the transformation function is

$$
\begin{aligned}
x & =t^{-1}(w) \\
& =\left\{\begin{array}{l}
k-w, x \leq k \\
w+k, x>k
\end{array}\right.
\end{aligned}
$$

Then

$$
\frac{d}{d w} t^{-1}(w)=\left\{\begin{array}{l}
-1, x \leq k \\
1, x>k
\end{array}\right.
$$

giving

$$
\left|\frac{d}{d w} t^{-1}(w)\right|=1
$$

for both regions of $X$. (Note that $w \geq 0$ for the range $x \leq k$ and $w>0$ for $x>k$.) This means that $h(w \mid C)$ is the sum of $f_{k}\left(t^{-1}(w) \mid C\right)$ for $x \leq k$ and $f_{k}\left(t^{-1}(w) \mid C\right)$ for $x>k$, which gives

$$
h(w \mid C)=\frac{f(k-w \mid N) P(N)+f(k+w \mid S) P(S)}{P_{k}(C)}, w \geq 0 .
$$


APPENDIX B (Continued)

Similarly,

$$
h(w \mid I)=\frac{f(k-w \mid S) \cdot P(S)+f(k+w \mid N) \cdot P(N)}{P_{k}(I)}, w \geq 0 .
$$

CoRollary 5: Maximum Percent Correct for the 1:X; 2:l $l_{k}(X)$ Task

The maximum probability of a correct decision attainable in the $1: X ; 2: l_{k}(X)$ task is equal to the maximum probability of a correct decision in the Type 1 task using $l(X)$ as the decision axis.

(The proof is given for the discrete case only.) To maximize percentage correct in either the 1:l(X) or the $1: X ; 2: l_{k}(X)$ task, the ratio of the prior probabilities for that task must be used as the criterion. For the 1:1 $(X)$ task, $P_{\beta}(C)$ is maximized using the criterion $\beta=P(N) / P(S)$; for the $1: X ; 2: l_{k}(X)$ task, the criterion on the $l_{k}(X)$ axis maximizing $P_{k, \gamma}\left(C_{2}\right)$ is $\gamma=P_{k}(I) / P_{k}(C)$, where $C_{2}$ is the event that the observer gets the Type 2 decision correct. For the Type 1 task in the discrete case, this means the maximum percentage correct, $P_{\beta}(C)_{\max }$, is achieved by saying "yes, signal" whenever

$$
\frac{P(X=x \mid S)}{P(X=x \mid N)}>\frac{P(N)}{P(S)} \text {. }
$$

Let all the elements $x$ for which $l(x)>P(N) / P(S)$ make up the set $A$; then, all the elements $x$ for which $l(x) \leq P(N) / P(S)$ make up $\bar{A}$. $P_{\beta}(C)_{\max }$ is given by

$$
P_{\beta}(C)_{\max }=\sum_{x \in A} P(X=x \mid S) \cdot P(S)+\sum_{x \in \bar{A}} P(X=x \mid N) \cdot P(N) .
$$

For a given $k, P_{k, \gamma}\left(C_{2}\right)_{\max }$ is achieved by saying "yes, correct" whenever

$$
\frac{P_{k}(X=x \mid C)}{P_{k}(X=x \mid I)}>\frac{P_{k}(I)}{P_{k}(C)}
$$

that is, when

and

$$
\frac{1}{l(x)} \cdot \frac{P(N)}{P(S)} \cdot \frac{P_{k}(I)}{P_{k}(C)}>\frac{P_{k}(I)}{P_{k}(C)}, \text { for } x \leq k \text { by Equation B8a }
$$

$$
l(x) \cdot \frac{P(S)}{P(N)} \cdot \frac{P_{k}(I)}{P_{k}(C)}>\frac{P_{k}(I)}{P_{k}(C)} \text {, for } x>k \text { by Equation B8b, }
$$

which is when

$$
l(x)<P(N) / P(S), \text { for } x \leq k
$$

and

$$
l(x)>P(N) / P(S), \text { for } x>k .
$$

The observer should, therefore, say "no, incorrect" in the Type 2 task when

$$
l(x) \geq P(N) / P(S), \text { for } x \leq k
$$

and

$$
l(x) \leq P(N) / P(S), \text { for } x>k .
$$

Let $B$ be the set of elements to which the observer responds "yes, correct" according to this decision rule. Then $B$ is made up of those elements $x \leq k$ for which $l(x)<P(N) / P(S)$ and those elements $x>k$ for which $l(x)>P(N) / P(S)$. The rest of the elements $x$ make up $\bar{B}$.

Consider the following six mutually exclusive sets, which combine to give the set of all possible values of $x$ :

$$
\begin{aligned}
& C=\{\text { elements } x \text { such that } x \leq k, l(x)<P(N) / P(S)\}, \\
& D=\{\text { elements } x \text { such that } x>k, l(x)<P(N) / P(S)\}, \\
& E=\{\text { elements } x \text { such that } x \leq k, l(x)=P(N) / P(S)\}, \\
& F=\{\text { elements } x \text { such that } x>k, l(x)=P(N) / P(S)\}, \\
& G=\{\text { elements } x \text { such that } x \leq k, l(x)>P(N) / P(S)\}, \\
& H=\{\text { elements } x \text { such that } x>k, l(x)>P(N) / P(S)\} .
\end{aligned}
$$




\begin{tabular}{|c|c|}
\hline$C$ & $D$ \\
$x \leq k$ & $x>k$ \\
$l(x)<\frac{P(N)}{P(S)}$ & $l(x)<\frac{P(N)}{P(S)}$ \\
\hline$E$ & $F$ \\
$x \leq k$ & $x>k$ \\
$l(x)=\frac{P(N)}{P(S)}$ & $l(x)=\frac{P(N)}{P(S)}$ \\
\hline$G$ & $H$ \\
$x \leq k$ & $x>k$ \\
$l(x)>\frac{P(N)}{P(S)}$ & $l(x)>\frac{P(N)}{P(S)}$ \\
\hline
\end{tabular}

Figure B2. Membership of $x$ values in Sets $A$ to $H$. The dashed line encloses sets belonging to $A$; the dotted line encloses sets belonging to $B$.

Then $A=G \cup H$ and $B=C \cup H$. These sets are diagrammed in Figure B2.

By the definitions of $B$ and $\bar{B}$, it can be seen that

$$
\begin{aligned}
P_{k, \gamma}\left(C_{2}\right)_{\max }= & \sum_{x \in \bar{B}} P_{k}(X=x \mid C) \cdot P_{k}(C)+\sum_{x \in \bar{B}} P_{k}(X=x \mid I) \cdot P_{k}(I) \\
= & \sum_{x \geq k, x \in B} P(X=x \mid N) \cdot P(N)+\sum_{x \geq k, x \in \bar{B}} P(X=x \mid S) \cdot P(S) \\
& +\sum_{x>k, x \in B} P(X=x \mid S) \cdot P(S)+\sum_{x>k, x \in \bar{B}} P(X=x \mid N) \cdot P(N) .
\end{aligned}
$$

Consider the elements of $\bar{B}$ which satisfy $x \leq k$ (i.e., those whose probabilities are summed in the second term of Equation B15). These elements belong to either $E$ or $G$. For those belonging to $E$, it is true that

or

$$
\begin{aligned}
& \frac{P(X=x \mid S)}{P(X=x \mid N)}>\frac{P(N)}{P(S)} \\
& P(X=x \mid N) \cdot P(N)=P(X=x \mid S) \cdot P(S),
\end{aligned}
$$

so the second term of the Equation B15 becomes

$$
\sum_{E \cup G} P(X=x \mid S) \cdot P(S)=\sum_{E} P(X=x \mid N) \cdot P(N)+\sum_{G} P(X=x \mid S) \cdot P(S) .
$$

Using the set labels in Figure B2, Equation B15 can be rewritten as

$$
\begin{aligned}
P_{k, \gamma}\left(C_{2}\right)_{\max }= & \sum_{C} P(X=x \mid N) \cdot P(N)+\sum_{E} P(X=x \mid N) \cdot P(N)+\sum_{G} P(X=x \mid S) \cdot P(S) \\
& +\sum_{H} P(X=x \mid S) \cdot P(S)+\sum_{E \cup F} P(X=x \mid N) \cdot P(N) \\
= & \sum_{G \cup H} P(X=x \mid S) \cdot P(S)+\sum_{C \cup D \cup E \cup F} P(X=x \mid N) \cdot P(N) \\
= & \sum_{A} P(X=x \mid S) \cdot P(S)+\sum_{\bar{A}} P(X=x \mid N) \cdot P(N) \quad \text { (seeFigureB2) } \\
= & P_{\beta}(C)_{\max } .
\end{aligned}
$$




\section{APPENDIX B (Continued)}

COROLLARY 6: Different Functions With the Same Type 2 ROC Curves

If two pairs of probability functions give the same Type 1 ROC curve, two criteria (one applied to each pair) that generate a common point on that ROC curve will each generate the same Type 2 ROC curve when applied to their respective probability functions.

Consider two pairs of probability functions $f_{1}(x \mid S), f_{1}(x \mid N)$ and $f_{2}(x \mid S), f_{2}(x \mid N)$ that have the same Type 1 ROC curve. For any criterion under the first pair of probability functions, there is a criterion under the second pair of probability functions that gives the same point on the ROC curve - that is, the same $H R$ and $F A R$. Therefore, there exists some function, $h$ such that

$$
1-F_{1}(x \mid N)=1-F_{2}[h(x) \mid N]
$$

and

$$
1-F_{1}(x \mid S)=1-F_{2}[h(x) \mid S]
$$

that is,

$$
F_{1}(x \mid N)=F_{2}[h(x) \mid N]
$$

and

$$
F_{1}(x \mid S)=F_{2}[h(x) \mid S] .
$$

Consider a criterion, $k_{1}$, under the first pair of probability functions and the criterion $k_{2}=h\left(k_{1}\right)$ under the second pair of probability functions that generates the same point on the Type 1 ROC curve. Let $\operatorname{ROC}_{2, k 1}$ be the Type 2 curve obtained using $k_{1}$ as the criterion on $f_{1}(x \mid S)$ and $f_{1}(x \mid N)$. Its coordinates are given by

$$
H R_{2, k_{1}}(x)= \begin{cases}\frac{P_{k_{1}}(C)-F_{1}(x \mid N) \cdot P(N)}{P_{k_{1}}(C)}, & \text { for } x \leq k_{1} \\ \frac{\left[1-F_{1}(x \mid S) \cdot P(S)\right]}{P_{k_{1}}(C)}, & \text { for } x>k_{1}\end{cases}
$$

and

$$
F A R_{2, k_{1}}(x)= \begin{cases}\frac{P_{k_{1}}(I)-F_{1}(x \mid S) \cdot P(S)}{P_{k_{1}}(I)}, & \text { for } x \leq k_{1} \\ \frac{\left[1-F_{1}(x \mid N) \cdot P(N)\right]}{P_{k_{1}}(I)}, & \text { for } x>k_{1},\end{cases}
$$

where

$$
P_{k_{1}}(C)=\left[1-F_{1}\left(k_{1} \mid S\right)\right] \cdot P(S)+F_{1}\left(k_{1} \mid N\right) \cdot P(N)
$$

and

$$
P_{k_{1}}(I)=F_{1}\left(k_{1} \mid S\right) \cdot P(S)+\left[1-F_{1}\left(k_{1} \mid N\right)\right] \cdot P(N) \text {. }
$$

The Type 2 curve based on the second pair of probability functions and using $k_{2}$ as criterion for the Type 1 decision is $\mathrm{ROC}_{2, k 2}$, given by

$$
H R_{2, k_{2}}(x)= \begin{cases}\frac{P_{k_{2}}(C)-F_{2}(x \mid N) \cdot P(N)}{P_{k_{1}}(C)}, & \text { for } x \leq k_{2} \\ \frac{\left[1-F_{2}(x \mid S)\right] \cdot P(S)}{P_{k_{2}}(C)}, & \text { for } x>k_{2}\end{cases}
$$

and

$$
F_{2, k_{1}}(x)= \begin{cases}\frac{P_{k_{2}}(I)-F_{2}(x \mid S) \cdot P(S)}{P_{k_{2}}(I)}, & \text { for } x \leq k_{2} \\ \frac{\left[1-F_{2}(x \mid N)\right] \cdot P(N)}{P_{k_{2}}(I)}, & \text { for } x>k_{2}\end{cases}
$$

where

$$
P_{k_{2}}(C)=\left[1-F_{2}\left(k_{2} \mid S\right)\right] \cdot P(S)+F_{2}\left(k_{2} \mid N\right) \cdot P(N)
$$

and

$$
P_{k_{2}}(I)=F_{2}\left(k_{2} \mid S\right) \cdot P(S)+\left[1-F_{2}\left(k_{2} \mid N\right)\right] \cdot P(N) .
$$




\section{APPENDIX B (Continued)}

Because $k_{1}$ and $k_{2}$ give the same point on the Type 1 curve when applied as criteria to their respective pairs of probability functions, it is true that

$$
1-F_{1}\left(k_{1} \mid S\right)=1-F_{2}\left(k_{2} \mid S\right)
$$

equating $H R$ s, and

$$
1-F_{1}\left(k_{1} \mid N\right)=1-F_{2}\left(k_{2} \mid N\right),
$$

equating FARs. Equations B18 to B23 togetherimply that, for the same pairs of prior probabilities,

$$
P_{k_{1}}(C)=P_{k_{2}}(C)
$$

and

$$
P_{k_{1}}(I)=P_{k_{2}}(I) \text {. }
$$

Now it can also be seen that the point on the $\mathrm{ROC}_{2, k 1}$ curve obtained when $k_{1}$ is used as the criterion in the Type 1 decision and then in the Type 2 decision is the same as the point on the $\mathrm{ROC}_{2, k 2}$ curve obtained using $k_{2}$ as the criterion for both decisions - that is,

$$
\frac{P_{k_{1}}(C)-F_{1}\left(k_{1} \mid N\right) \cdot P(N)}{P_{k_{1}}(C)}=\frac{P_{k_{2}}(C)-F_{2}\left(k_{2} \mid N\right) \cdot P(N)}{P_{k_{2}}(C)}
$$

equating $H R$ s, and

$$
\frac{P_{k_{1}}(I)-F_{1}\left(k_{1} \mid S\right) \cdot P(S)}{P_{k_{1}}(I)}=\frac{P_{k_{2}}(I)-F_{2}\left(k_{2} \mid S\right) \cdot P(S)}{P_{k_{2}}(I)},
$$

equating FARs.

Finally, the stretches of the Type 2 ROC curve above and below this point can be shown to be the same, as follows. The inequality $\mathrm{x} \leq k_{1}$ is true if and only if

$$
1-F_{1}(x \mid N) \geq 1-F_{1}\left(k_{1} \mid N\right)
$$

that is,

$$
1-F_{2}[h(x) \mid N] \geq 1-F_{2}\left(k_{2} \mid N\right), \text { by Equations B16 and B17, }
$$

which gives

$$
h(x) \geq k_{2} .
$$

Substituting $h(x)$ for $x$ in the formulae for $\mathrm{ROC}_{2, k 2}$ gives

$$
H R_{2, k_{2}}[h(x)]= \begin{cases}\frac{P_{k_{2}}(C)-F_{2}[h(x) \mid N] \cdot P(N)}{P_{k_{2}}(C)}, & \text { for } h(x) \leq k_{2} \\ \frac{\left[1-F_{2}[h(x) \mid S] \cdot P(S)\right]}{P_{k_{2}}(C)}, & \text { for } h(x)>k_{2}\end{cases}
$$

and

$$
F A R_{2, k_{1}}[h(x)]= \begin{cases}\frac{P_{k_{2}}(I)-F_{2}[h(x) \mid S] \cdot P(S)}{P_{k_{2}}(I)}, & \text { for } h(x) \leq k_{2} \\ \frac{\left[1-F_{2}[h(x) \mid N] \cdot P(N)\right]}{P_{k_{2}}(I)}, & \text { for } h(x)>k_{2}\end{cases}
$$

which, by the equalities B16, B17, B24, and B25 and the inequality B26, are identical to the equations for the $\mathrm{ROC}_{2, k 1}$ curve. This means that just as $x$ and $h(x)$ gave the same point on the Type 1 curve when applied to the $f_{1}$ and $f_{2}$ functions, respectively, $x$ yields the same point on the $\mathrm{ROC}_{2, k 1}$ curve as $h(x)$ yields on the $\mathrm{ROC}_{2, k 2}$ curve when $x$ and $h(x)$ are used as criteria on the functions underlying these two curves, respectively. 